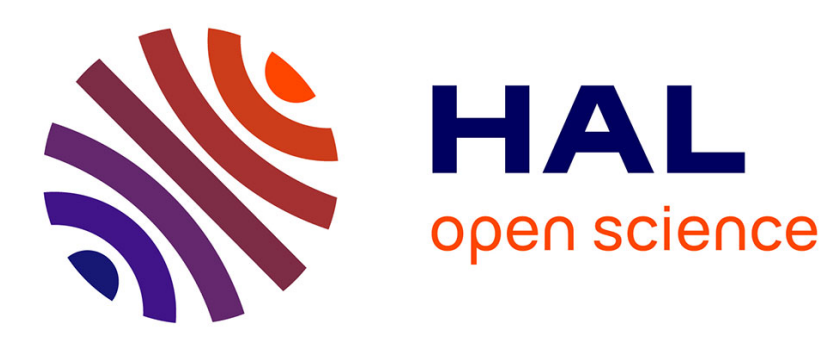

\title{
Stochastic model specification search for Gaussian and partial non-Gaussian state space models
}

Sylvia Frühwirth-Schnatter, Helga Wagner

\section{To cite this version:}

Sylvia Frühwirth-Schnatter, Helga Wagner. Stochastic model specification search for Gaussian and partial non-Gaussian state space models. Econometrics, 2009, 154 (1), pp.85. 10.1016/j.jeconom.2009.07.003 . hal-00610792

\section{HAL Id: hal-00610792 https://hal.science/hal-00610792}

Submitted on 25 Jul 2011

HAL is a multi-disciplinary open access archive for the deposit and dissemination of scientific research documents, whether they are published or not. The documents may come from teaching and research institutions in France or abroad, or from public or private research centers.
L'archive ouverte pluridisciplinaire HAL, est destinée au dépôt et à la diffusion de documents scientifiques de niveau recherche, publiés ou non, émanant des établissements d'enseignement et de recherche français ou étrangers, des laboratoires publics ou privés. 


\section{Accepted Manuscript}

Stochastic model specification search for Gaussian and partial

non-Gaussian state space models

Sylvia Frühwirth-Schnatter, Helga Wagner

PII:

S0304-4076(09)00161-4

DOI:

10.1016/j.jeconom.2009.07.003

Reference: ECONOM 3218

To appear in: Journal of Econometrics

Received date: 23 May 2008

Revised date: 2 July 2009

Accepted date: 19 July 2009

Please cite this article as: Frühwirth-Schnatter, S., Wagner, H., Stochastic model specification search for Gaussian and partial non-Gaussian state space models. Journal of Econometrics (2009), doi:10.1016/j.jeconom.2009.07.003

This is a PDF file of an unedited manuscript that has been accepted for publication. As a service to our customers we are providing this early version of the manuscript. The manuscript will undergo copyediting, typesetting, and review of the resulting proof before it is published in its final form. Please note that during the production process errors may be discovered which could affect the content, and all legal disclaimers that apply to the journal pertain. 


\title{
Stochastic Model Specification Search for Gaussian and Partial Non-Gaussian State Space Models
}

\author{
Sylvia Frühwirth-Schnatter ${ }^{1}$ and Helga Wagner \\ Department of Applied Statistics and Econometrics, Johannes Kepler Universität Linz, \\ Austria
}

ABSTRACT

Model specification for state space models is a difficult task as one has to decide which components to include in the model and to specify whether these components are fixed or timevarying. To this aim a new model space MCMC method is developed in this paper. It is based on extending the Bayesian variable selection approach which is usually applied to variable selection in regression models to state space models. For non-Gaussian state space models stochastic model search MCMC makes use of auxiliary mixture sampling. We focus on structural time series models including seasonal components, trend or intervention. The method is applied to various well-known time series.

Key words: auxiliary mixture sampling, Bayesian econometrics, noncentered parameterization, Markov chain Monte Carlo, variable selection

\footnotetext{
${ }^{1}$ Altenbergerstraße 69, A-4040 Linz, Austria; Phone: ++43 7322468 8295; e-mail: sylvia.fruehwirthschnatter@jku.at
} 


\section{Introduction}

State space models are widely used in time series analysis to deal with processes which gradually change over time. Model specification, however, is a challenge for these models as one has to specify which components to include and to decide whether they are fixed or time-varying. For state space models, like for many other complex models, this often leads to testing problems which are non-regular from the view-point of classical statistics. Thus, a classical approach toward model selection which is based on hypothesis testing such as a likelihood ratio test or information criteria such as AIC or BIC cannot be easily applied, because it relies on asymptotic arguments based on regularity conditions that are violated in this context.

Consider, for example, modeling a time series $\mathbf{y}=\left(y_{1}, \ldots, y_{T}\right)$ through the dynamic linear trend model, defined for $t=1, \ldots, T$ as:

$$
y_{t}=\mu_{t}+\varepsilon_{t}, \quad \varepsilon_{t} \sim \mathcal{N}\left(0, \sigma_{\varepsilon}^{2}\right)
$$

where $\mu_{t}$ follows a random walk with a random drift starting from unknown initial values $\mu_{0}$ and $a_{0}$ :

$$
\begin{aligned}
& \mu_{t}=\mu_{t-1}+a_{t-1}+\omega_{1 t}, \quad \omega_{1 t} \sim \mathcal{N}\left(0, \theta_{1}\right) \\
& a_{t}=a_{t-1}+\omega_{2 t}, \quad \omega_{2 t} \sim \mathcal{N}\left(0, \theta_{2}\right)
\end{aligned}
$$

A typical specification problem arising for this model is to decide if the drift $a_{t}$ is timevarying rather than constant. However, testing $\theta_{2}=0$ versus $\theta_{2}>0$ results in a nonregular testing problem, because the null hypothesis lies on the boundary of the parameter space. A similar specification problem is deciding which components are present in this times series model. For instance, is it necessary to include a dynamic drift term $a_{t}$ or should $a_{t}$ be removed because the level $\mu_{t}$ follows a simple random walk? This is another non-regular problem, because again the null hypothesis can be rephrased as testing $a_{0}=\theta_{2}=0$.

The Bayesian approach is, in principle, able to deal with such non-regular testing problems. Suppose that $K$ different models $\mathcal{M}_{1}, \ldots, \mathcal{M}_{K}$ are considered to be candidates for having generated the time series $\mathbf{y}$. In a Bayesian setting each of these models is assigned a prior probability $p\left(\mathcal{M}_{k}\right)$ and the goal is to derive the posterior model probability $p\left(\mathcal{M}_{k} \mid \mathbf{y}\right)$ for each model $\mathcal{M}_{k}, k=1, \ldots, K$. 
There are basically two strategies to cope with the challenge associated with computing the posterior model probabilities. The traditional approach dating back to Jeffreys (1948) and Zellner (1971) determines the posterior model probabilities of each model separately by using Bayes' rule, $p\left(\mathcal{M}_{k} \mid \mathbf{y}\right) \propto p\left(\mathbf{y} \mid \mathcal{M}_{k}\right) p\left(\mathcal{M}_{k}\right)$, where $p\left(\mathbf{y} \mid \mathcal{M}_{k}\right)$ is the marginal likelihood for model $\mathcal{M}_{k}$. An explicit expression for the marginal likelihood exists only for conjugate problems like linear regression models with normally distributed errors, whereas for more complex models numerical techniques are required. For Gaussian state space models, marginal likelihoods have been estimated using methods such as importance sampling (Frühwirth-Schnatter, 1995; Durbin and Koopman, 2000), Chib's estimator (Chib, 1995), numerical integration (Shively and Kohn, 1997) and bridge sampling (Frühwirth-Schnatter, 2001). Recently, Frühwirth-Schnatter and Wagner (2008) considered estimation of the marginal likelihood for non-Gaussian state space models and demonstrated that the resulting estimators can be pretty inaccurate.

The modern approach to Bayesian model selection is to apply model space MCMC methods by sampling jointly model indicators and parameters, using e.g. the reversible jump MCMC algorithm (Green, 1995) or the stochastic variable selection approach (George and McCulloch, 1993, 1997). The stochastic variable selection approach is commonly applied to model selection for regression models and aims at identifying non-zero regression effects, but it is useful far beyond this problem. It allows parsimonious covariance modelling for longitudinal data as shown by Smith and Kohn (2002) and covariance selection in random effects models as shown by Chen and Dunson (2003) and Frühwirth-Schnatter and Tüchler (2008).

Shively, Kohn, and Wood (1999) present a variable selection approach to non-parametric regression using priors for the unknown functions that are expressed in state space form, however, they did not deal explicitly with time series models. In the present paper we show that such a variable selection approach is useful for dealing with model selection problems in more general state space models.

To perform stochastic model specification search for the dynamic linear trend model defined in (1) to (3), for instance, we introduce three binary stochastic indicators in such a way that the unconstrained model corresponds to setting all indicators equal to 1 . Reduced model specifications result by setting certain indicators equal to 0 . One of those models, for instance, is the local level model, where the drift component $a_{t}$ completely 
disappears:

$$
\mu_{t}=\mu_{t-1}+\omega_{1 t}, \quad \omega_{1 t} \sim \mathcal{N}\left(0, \theta_{1}\right)
$$

Another interesting special case is the linear trend model, where

$$
y_{t}=\mu_{0}+t a_{0}+\varepsilon_{t}, \quad \varepsilon_{t} \sim \mathcal{N}\left(0, \sigma_{\varepsilon}^{2}\right) .
$$

The practical implementation of this approach is innovative in two respects. First, we employ a new prior for the process variances of the state space model by assuming that the square root of each process variance follows a normal distribution centered at 0 . It is well-known that variable selection is, in general, sensitive to the choice of the prior, see e.g. Fernández, Ley, and Steel (2001). We show both for simulated as well as for real data that this prior is less influential on posterior inference when the true process variance is close to 0 than the usually applied inverted Gamma prior. This is in line with Gelman (2006) who came to similar conclusions for the related random-effects model.

Second, we derive an MCMC method for Gaussian as well as partially Gaussian state space models that performs stochastic model specification search by sampling the indicators simultaneously with the models parameters. The sampler is based on a noncentered parameterization of the state space model which generalizes previous work in this area such as Pitt and Shephard (1999) and Frühwirth-Schnatter (2004). In combination with the normal prior on the square root of each process variance this leads to Gibbs sampler that is easily implemented. This is in contrast to Shively et al. (1999) who consider a data-based prior which is motivated by the BIC criterion and leads to a sampling scheme where numerical integration has to be performed for each sweep of the MCMC scheme in order to sample indicators and parameters jointly.

To implement this approach for non-Gaussian state space modeling of times series of count data or binary, categorical, or multinomial data we make use of auxiliary mixture sampling (Frühwirth-Schnatter and Wagner, 2006; Frühwirth-Schnatter and Frühwirth, 2007; Frühwirth-Schnatter, Frühwirth, Held, and Rue, 2009) which is a simple MCMC method for estimating a broad class of discrete-valued models.

Throughout the paper we focus on structural time series models including seasonal components, trend and an intervention effect and apply the method to various well-known time series. 


\section{The Dynamic Linear Trend Model}

Our method is based on a noncentered parameterization of the dynamic linear trend model which is discussed in the next subsection.

\subsection{A Noncentered Parameterization}

Define two independent random walk processes $\tilde{\mu}_{t}$ and $\tilde{a}_{t}$ with standard normal independent increments as well as an integrated process $\tilde{A}_{t}$ :

$$
\begin{aligned}
\tilde{\mu}_{t} & =\tilde{\mu}_{t-1}+\tilde{\omega}_{1 t}, & \tilde{\omega}_{1 t} \sim \mathcal{N}(0,1), \\
\tilde{a}_{t} & =\tilde{a}_{t-1}+\tilde{\omega}_{2 t}, & \tilde{\omega}_{2 t} \sim \mathcal{N}(0,1), \\
\tilde{A}_{t} & =\tilde{A}_{t-1}+\tilde{a}_{t-1}, &
\end{aligned}
$$

which all are assumed to start at zero: $\tilde{\mu}_{0}=\tilde{a}_{0}=\tilde{A}_{0}=0$. Combine the state equations (6) to (7) with following observation equation:

$$
y_{t}=\mu_{0}+t a_{0}+\sqrt{\theta_{1}} \tilde{\mu}_{t}+\sqrt{\theta_{2}} \tilde{A}_{t}+\varepsilon_{t}, \quad \varepsilon_{t} \sim \mathcal{N}\left(0, \sigma_{\varepsilon}^{2}\right)
$$

where $\mu_{0}$ and $a_{0}$ are equal to the initial values for the level and the drift component and $\theta_{1}$ and $\theta_{2}$ are equal to the variances in the dynamic linear trend model defined in (1) to (3). The resulting state space model is a noncentered parameterization of the dynamic linear trend model. To verify this define

$$
\begin{aligned}
& a_{t}=a_{0}+\sqrt{\theta_{2}} \tilde{a}_{t}, \\
& \mu_{t}=\mu_{0}+t a_{0}+\sqrt{\theta_{1}} \tilde{\mu}_{t}+\sqrt{\theta_{2}} \tilde{A}_{t} .
\end{aligned}
$$

Then

$$
\begin{aligned}
a_{t}-a_{t-1} & =\sqrt{\theta_{2}}\left(\tilde{a}_{t}-\tilde{a}_{t-1}\right)=\sqrt{\theta_{2}} \tilde{\omega}_{2 t}=\omega_{2 t}, \quad \omega_{2 t} \sim \mathcal{N}\left(0, \theta_{2}\right), \\
\mu_{t}-\mu_{t-1} & =\sqrt{\theta_{1}}\left(\tilde{\mu}_{t}-\tilde{\mu}_{t-1}\right)+a_{0}+\sqrt{\theta_{2}}\left(\tilde{A}_{t}-\tilde{A}_{t-1}\right) \\
& =\sqrt{\theta_{1}} \tilde{\omega}_{1 t}+a_{0}+\sqrt{\theta_{2}} \tilde{a}_{t-1}=\omega_{1 t}+a_{t-1}, \quad \omega_{1 t} \sim \mathcal{N}\left(0, \theta_{1}\right),
\end{aligned}
$$

which corresponds to the state equations (2) and (3). 
The noncentered parameterization of the dynamic linear trend model has a representation as a state space model with a state vector of dimension 3 :

$$
\begin{aligned}
& \mathbf{x}_{t}=\mathbf{F x}_{t-1}+\mathbf{w}_{t}, \quad \mathbf{w}_{t} \sim \mathcal{N}(\mathbf{0}, \mathbf{Q}), \\
& y_{t}=\mathbf{H x}_{t}+\boldsymbol{z}_{t}^{f} \boldsymbol{\alpha}+\varepsilon_{t}, \quad \varepsilon_{t} \sim \mathcal{N}\left(0, \sigma_{\varepsilon}^{2}\right),
\end{aligned}
$$

where $\mathbf{x}_{0}=\mathbf{0}_{3 \times 1}$ and

$$
\begin{aligned}
& \mathbf{x}_{t}=\left(\begin{array}{c}
\tilde{\mu}_{t} \\
\tilde{a}_{t} \\
\tilde{A}_{t}
\end{array}\right), \quad \mathbf{F}=\left(\begin{array}{ccc}
1 & 0 & 0 \\
0 & 1 & 0 \\
0 & 1 & 1
\end{array}\right), \quad \mathbf{Q}=\left(\begin{array}{lll}
1 & 0 & 0 \\
0 & 1 & 0 \\
0 & 0 & 0
\end{array}\right), \\
& \mathbf{H}=\left(\begin{array}{lll}
\sqrt{\theta_{1}} & 0 & \sqrt{\theta_{2}}
\end{array}\right), \quad \boldsymbol{z}_{t}^{f}=\left(\begin{array}{ll}
1 & t
\end{array}\right), \quad \boldsymbol{\alpha}=\left(\begin{array}{ll}
\mu_{0} & a_{0}
\end{array}\right)^{\prime} .
\end{aligned}
$$

This state space form could be used to perform Kalman filtering and to compute the integrated likelihood $p(\mathbf{y} \mid \boldsymbol{\vartheta})$ for $\boldsymbol{\vartheta}=\left(\sqrt{\theta_{1}}, \sqrt{\theta_{2}}, \sigma_{\varepsilon}^{2}, \mu_{0}, a_{0}\right)$.

The noncentered parameterization of the dynamic linear trend model, however, is not identified, because in the observation equation (8), the sign of $\sqrt{\theta_{1}}$ and the sequence $\left\{\tilde{\mu}_{t}\right\}_{1}^{T}$ may be changed by multiplying all elements with -1 without changing the distribution of $y_{1}, \ldots, y_{T}$. If we define a state vector $\mathbf{x}_{t}^{\star}=\left(-\tilde{\mu}_{t}, \tilde{a}_{t}, \tilde{A}_{t}\right)^{\prime}$ and a parameter $\boldsymbol{\vartheta}^{\star}=\left(-\sqrt{\theta_{1}}, \sqrt{\theta_{2}}, \sigma_{\varepsilon}^{2}, \mu_{0}, a_{0}\right)$, then $\boldsymbol{\vartheta}^{\star}$ and $\boldsymbol{\vartheta}$, although being different, define the same integrated likelihood:

$$
\begin{aligned}
& p(\mathbf{y} \mid \boldsymbol{\vartheta})=\int p\left(\mathbf{y} \mid \mathbf{x}_{1}, \ldots, \mathbf{x}_{T}, \sqrt{\theta_{1}}, \sqrt{\theta_{2}}, \sigma_{\varepsilon}^{2}, \mu_{0}, a_{0}\right) p\left(\mathbf{x}_{1}, \ldots, \mathbf{x}_{T}\right) d\left(\mathbf{x}_{1}, \ldots, \mathbf{x}_{T}\right) \\
& =\int p\left(\mathbf{y} \mid \mathbf{x}_{1}^{\star}, \ldots, \mathbf{x}_{T}^{\star},-\sqrt{\theta_{1}}, \sqrt{\theta_{2}}, \sigma_{\varepsilon}^{2}, \mu_{0}, a_{0}\right) p\left(\mathbf{x}_{1}^{\star}, \ldots, \mathbf{x}_{T}^{\star}\right) d\left(\mathbf{x}_{1}^{\star}, \ldots, \mathbf{x}_{T}^{\star}\right)=p\left(\mathbf{y} \mid \boldsymbol{\vartheta}^{\star}\right) .
\end{aligned}
$$

Similarly, the sign of $\sqrt{\theta_{2}}$ and the sequences $\left\{\tilde{a}_{t}\right\}_{1}^{T}$ and $\left\{\tilde{A}_{t}\right\}_{1}^{T}$ may be changed without changing the distribution of $y_{1}, \ldots, y_{T}$ and $\boldsymbol{\vartheta}^{\star}=\left(\sqrt{\theta_{1}},-\sqrt{\theta_{2}}, \sigma_{\varepsilon}^{2}, \mu_{0}, a_{0}\right)$ and $\boldsymbol{\vartheta}$ define the same integrated likelihood, $p(\mathbf{y} \mid \boldsymbol{\vartheta})=p\left(\mathbf{y} \mid \boldsymbol{\vartheta}^{\star}\right)$.

As a consequence, the likelihood function $p(\mathbf{y} \mid \boldsymbol{\vartheta})$ is symmetric around 0 in the direction of $\sqrt{\theta_{1}}$ and $\sqrt{\theta_{2}}$ and therefore multimodal. If the data are generated by a dynamic linear trend model with true parameters $\left(\theta_{1}^{\operatorname{tr}}, \theta_{2}^{\operatorname{tr}}, \boldsymbol{\xi}^{\operatorname{tr}}\right)$, where $\boldsymbol{\xi}^{\operatorname{tr}}=\left(\sigma_{\varepsilon}^{2, \operatorname{tr}}, \mu_{0}^{\operatorname{tr}}, a_{0}^{\operatorname{tr}}\right)$, then with increasing number of observations $T$, the modes of the likelihood function will be close to $\left(\sqrt{\theta_{1}^{\operatorname{tr}}}, \sqrt{\theta_{2}^{\operatorname{tr}}}, \boldsymbol{\xi}^{\mathrm{tr}}\right),\left(-\sqrt{\theta_{1}^{\operatorname{tr}}}, \sqrt{\theta_{2}^{\operatorname{tr}}}, \boldsymbol{\xi}^{\mathrm{tr}}\right),\left(\sqrt{\theta_{1}^{\operatorname{tr}}},-\sqrt{\theta_{2}^{\operatorname{tr}}}, \boldsymbol{\xi}^{\mathrm{tr}}\right)$, and $\left(-\sqrt{\theta_{1}^{\operatorname{tr}}},-\sqrt{\theta_{2}^{\operatorname{tr}}}, \boldsymbol{\xi}^{\mathrm{tr}}\right)$. If the true variances $\theta_{1}^{\text {tr }}$ and $\theta_{2}^{\text {tr }}$ are positive, then the likelihood function concentrates around 
four modes. If one the true variances is equal to 0 while the other is positive, two of those modes collapse and the likelihood is bimodal with increasing $T$. If both variances $\theta_{1}^{\operatorname{tr}}$ and $\theta_{2}^{\operatorname{tr}}$ are equal to zero, then the likelihood function becomes unimodal as $T$ increases.

For illustration, Figure 1 shows contour and surface plots of the (scaled) likelihood function $p\left(\mathbf{y} \mid \sqrt{\theta_{1}}, \sqrt{\theta_{2}}, \sigma_{\varepsilon}^{2, \operatorname{tr}}, \mu_{0}^{\operatorname{tr}}, a_{0}^{\operatorname{tr}}\right)$ for a time series of length $T=1000$ simulated from a dynamic linear trend model with $\mu_{0}^{\text {tr }}=0.3, a_{0}^{\text {tr }}=-0.1$ and $\sigma_{\varepsilon}^{2, \text { tr }}=1$ and four different combinations of $\theta_{1}^{\text {tr }}$ and $\theta_{2}^{\text {tr }}$. There are clearly four modes, if both process variances are positive, two modes, if one of the variances is restricted to zero and a single mode, if both variances are restricted to 0 .

Thus by considering the non-centered parameterization and allowing for nonidentifiability we gain important information about the hypothesis whether the variances of the state space model are zero.

\subsection{The Parsimonious Dynamic Linear Trend Model}

The noncentered parameterization of the dynamic linear trend model is very useful for model selection both for the components and the dynamics. The observation equation (8) of the noncentered parameterization represents the level of the time series $y_{t}$ as a superposition of the components at time $t=0$ and the random processes $\tilde{\mu}_{t}$ and $\tilde{A}_{t}$. Note that neither $\tilde{\mu}_{t}$ nor $\tilde{A}_{t}$ degenerate to a static component. A static component is obtained by setting the appropriate variance equal to 0 . For instance, if the variance $\theta_{1}$ is equal to 0 , then $\sqrt{\theta_{1}}=0$ and $\tilde{\mu}_{t}$ is not used to explain $y_{t}$. Similarly, if the variance $\theta_{2}$ is equal to 0 , then $\sqrt{\theta_{2}}=0$ and $\tilde{A}_{t}$ is not used to explain $y_{t}$. This suggests to consider the choice of the variances $\theta_{1}$ and $\theta_{2}$ as a variable selection problem in regression model (8).

To this aim we introduce two binary indicators $\gamma_{1}$ and $\gamma_{2}$, where $\sqrt{\theta_{i}}$, and consequently $\theta_{i}$, is equal to 0 , if $\gamma_{i}=0$. If $\gamma_{i}=1$, then $\sqrt{\theta_{i}}$ is an unconstrained unknown parameter which is estimated from the data under a suitable prior. Evidently, the indicators $\gamma_{1}$ and $\gamma_{2}$ decide if a certain component of the state vector is fixed or changes over time. If both $\gamma_{1}=0$ and $\gamma_{2}=0$, then the model reduces to a regression model with a linear trend, given by (5).

To include or delete the trend, an additional indicator $\delta$ is introduced which decides, if the initial slope $a_{0}$ is equal to 0 or not. If $\delta=0$, then $a_{0}$ is equal to 0 ; otherwise, if $\delta=1$, 
then $a_{0}$ is an unknown parameter which is estimated from the data under a suitable prior. This leads to the following parsimonious dynamic linear trend model:

$$
\begin{aligned}
\tilde{\mu}_{t} & =\tilde{\mu}_{t-1}+\tilde{\omega}_{1 t}, \quad \tilde{\omega}_{1 t} \sim \mathcal{N}(0,1), \\
\tilde{a}_{t} & =\tilde{a}_{t-1}+\tilde{\omega}_{2 t}, \quad \tilde{\omega}_{2 t} \sim \mathcal{N}(0,1), \\
\tilde{A}_{t} & =\tilde{a}_{t-1}+\tilde{A}_{t-1}, \\
y_{t} & =\mu_{0}+\delta t a_{0}+\gamma_{1} \sqrt{\theta_{1}} \tilde{\mu}_{t}+\gamma_{2} \sqrt{\theta_{2}} \tilde{A}_{t}+\varepsilon_{t}, \quad \varepsilon_{t} \sim \mathcal{N}\left(0, \sigma_{\varepsilon}^{2}\right) .
\end{aligned}
$$

For a direct comparison with the usual dynamic linear trend model it is useful to rewrite the parsimonious model in the centered parameterization. Define

$$
\begin{aligned}
& a_{t}=\delta a_{0}+\sqrt{\theta_{2}} \tilde{a}_{t} \\
& \mu_{t}=\mu_{0}+\delta t a_{0}+\gamma_{1} \sqrt{\theta_{1}} \tilde{\mu}_{t}+\gamma_{2} \sqrt{\theta_{2}} \tilde{A}_{t} .
\end{aligned}
$$

Then (11) to (14) may be rewritten as:

$$
\begin{aligned}
& \mu_{t}=\mu_{t-1}+\delta a_{0}+\gamma_{2}\left(a_{t-1}-\delta a_{0}\right)+\gamma_{1} \omega_{1 t}, \quad \omega_{1 t} \sim \mathcal{N}\left(0, \theta_{1}\right), \\
& a_{t}=a_{t-1}+\omega_{2 t}, \quad \omega_{2 t} \sim \mathcal{N}\left(0, \theta_{2}\right) \\
& y_{t}=\mu_{t}+\varepsilon_{t}, \quad \varepsilon_{t} \sim \mathcal{N}\left(0, \sigma_{\varepsilon}^{2}\right) .
\end{aligned}
$$

Evidently, $\left(\delta, \gamma_{1}, \gamma_{2}\right)=(1,1,1)$ corresponds to the unrestricted dynamic linear trend model (2). The combination $\left(\delta, \gamma_{1}, \gamma_{2}\right)=(0,1,0)$ leads to the local level model (4) which is also known as exponential smoothing, the combination $\left(\delta, \gamma_{1}, \gamma_{2}\right)=(1,0,0)$ leads to a regression model with a deterministic linear trend, given by $(5)$ and $\left(\delta, \gamma_{1}, \gamma_{2}\right)=(0,0,0)$ leads to i.i.d. normal data, $y_{t} \sim \mathcal{N}\left(\mu_{0}, \sigma_{\varepsilon}^{2}\right)$.

The indicators $\delta, \gamma_{1}$ and $\gamma_{2}$ have to be introduced carefully into the centered parametrization. Consider the following alternative choice which appears more natural than (17) and (18), but leads to nonindentifiability:

$$
\begin{aligned}
& \mu_{t}=\mu_{t-1}+\delta a_{t-1}+\gamma_{1} \omega_{1 t}, \quad \omega_{1 t} \sim \mathcal{N}\left(0, \theta_{1}\right), \\
& a_{t}=a_{t-1}+\gamma_{2} \omega_{2 t}, \quad \omega_{2 t} \sim \mathcal{N}\left(0, \theta_{2}\right) .
\end{aligned}
$$

After recursive substitution we get following representation of the model as a normal linear mixed model:

$$
y_{t}=\mu_{0}+\delta t a_{0}+\gamma_{1} \sum_{j=1}^{t} \omega_{1 j}+\delta \gamma_{2} \sum_{j=1}^{t-1}(t-j) \omega_{2 j}+\varepsilon_{t},
$$


with fixed effects $\left(\mu_{0}, a_{0}\right)$ and random effects $\left(\omega_{1 j}, \omega_{2 j}\right), j=1 \ldots, t$. Only 6 models among the 8 possible combinations of the indicators $\left(\delta, \gamma_{1}, \gamma_{2}\right)$ are identifiable, because $\gamma_{2}$ is not identified, if $\delta=0$. In contrast to that, model (17) to (19) has the representation

$$
y_{t}=\mu_{0}+\delta t a_{0}+\gamma_{1} \sum_{j=1}^{t} \omega_{1 j}+\gamma_{2} \sum_{j=1}^{t-1}(t-j) \omega_{2 j}+\varepsilon_{t} .
$$

Evidently, all 8 combinations of the indicators $\left(\delta, \gamma_{1}, \gamma_{2}\right)$ are identifiable.

The noncentered parameterization of the parsimonious dynamic linear trend model given by (11) to (14) has the following representation as a state space model:

$$
\begin{aligned}
& \mathbf{x}_{t}=\mathbf{F} \mathbf{x}_{t-1}+\mathbf{w}_{t}, \quad \mathbf{w}_{t} \sim \mathcal{N}(\mathbf{0}, \mathbf{Q}), \\
& y_{t}=\mathbf{H}\left(\gamma_{1}, \gamma_{2}\right) \mathbf{x}_{t}+\boldsymbol{z}_{t}^{f}(\delta) \boldsymbol{\alpha}+\varepsilon_{t}, \quad \varepsilon_{t} \sim \mathcal{N}\left(0, \sigma_{\varepsilon}^{2}\right),
\end{aligned}
$$

where $\mathbf{x}_{t}, \mathbf{F}, \mathbf{Q}$ and $\boldsymbol{\alpha}$ are the same as in (9), while $\mathbf{H}$ and $\boldsymbol{z}_{t}^{f}$ depend on the model indicators:

$$
\mathbf{H}\left(\gamma_{1}, \gamma_{2}\right)=\left(\begin{array}{lll}
\gamma_{1} \sqrt{\theta_{1}} & 0 & \gamma_{2} \sqrt{\theta_{2}}
\end{array}\right), \quad \boldsymbol{z}_{t}^{f}(\delta)=\left(\begin{array}{cc}
1 & \delta t
\end{array}\right)
$$

\subsection{Prior Distributions}

To perform Bayesian estimation one has to choose a prior distribution $p\left(\delta, \gamma_{1}, \gamma_{2}\right)$ for all possible combinations of indicators. Subsequently, we assume a uniform distribution over all 8 combinations of the indicators. A more flexible distribution is discussed in Section 5 .

As common for dynamic linear trend models, we assume that apriori $\mu_{0}$ and $a_{0}$ are independently normally distributed, $\mu_{0} \sim \mathcal{N}\left(y_{1}, P_{0,11} \sigma_{\varepsilon}^{2}\right)$ and $a_{0} \sim \mathcal{N}\left(0, P_{0,22} \sigma_{\varepsilon}^{2}\right)$. Furthermore we assume an inverted Gamma prior $\mathcal{G}^{-1}\left(c_{0}, C_{0}\right)$ for the observation variance $\sigma_{\varepsilon}^{2}$

In contrast to previous work, we do not use the usual inverted Gamma priors $\theta_{1} \sim$ $\mathcal{G}^{-1}\left(d_{0,1}, D_{0,1}\right)$ and $\theta_{2} \sim \mathcal{G}^{-1}\left(d_{0,2}, D_{0,2}\right)$. We employ a new prior for the process variances of the state space model by assuming that the square root of each process variance follows a normal distribution centered at 0 , i.e. $\sqrt{\theta_{1}} \sim \mathcal{N}\left(0, B_{0,1} \sigma_{\varepsilon}^{2}\right)$ and $\sqrt{\theta_{2}} \sim \mathcal{N}\left(0, B_{0,2} \sigma_{\varepsilon}^{2}\right)$. It be should noted that the two priors are equivalent only under the limiting case of 
following improper priors: an inverted Gamma prior where $d_{0, i}=-0.5$ and $D_{0, i}=0$, i.e. $p\left(\theta_{i}\right) \propto \sqrt{\theta_{i}}$, and a normal prior where $B_{0, i}^{-1}=0$, i.e. $p\left(\sqrt{\theta_{i}}\right) \propto$ constant.

It is well-known, that the hyperparameters of the inverted Gamma prior $\theta_{i} \sim \mathcal{G}^{-1}\left(d_{0, i}, D_{0, i}\right)$ strongly influence the posterior density of $\theta_{i}$, if the true value of $\theta_{i}$ is close to 0 . In contrast to that, the normal prior appears to be less influential and more suitable under model specification uncertainty than the inverted Gamma prior.

Consider, for example, a local level model,

$$
\begin{aligned}
& \mu_{t}=\mu_{t-1}+\omega_{1 t}, \quad \omega_{1 t} \sim \mathcal{N}\left(0, \theta_{1}\right), \\
& y_{t}=\mu_{t}+\varepsilon_{t}, \quad \varepsilon_{t} \sim \mathcal{N}\left(0, \sigma_{\varepsilon}^{2}\right),
\end{aligned}
$$

where $\theta_{1}$ is unknown and $\sigma_{\varepsilon}^{2}$ is assumed to be known.

To compare the inverted Gamma prior to the normal prior we consider the posterior density of the parameter $\pm \sqrt{\theta_{1}}$ which is obtained from $\theta_{1}$ by multiplying the square root of $\theta_{1}$ with a random sign. We added the \pm sign to emphasize that the sign of this parameter is not identified. The posterior of $\pm \sqrt{\theta_{1}}$ allows to explore the hypothesis that $\theta_{1}=0$. Due to the symmetry of the likelihood discussed in Subsection 2.1, the posterior density of $\pm \sqrt{\theta_{1}}$ is symmetric around zero as long as the prior is also symmetric around 0 . If the unknown variance $\theta_{1}^{\text {tr }}$ is significantly different from zero, then the posterior density of $\pm \sqrt{\theta_{1}}$ is likely to be bimodal with the modes being close to $\pm \sqrt{\theta_{1}^{\operatorname{tr}}}$. Otherwise, if $\theta_{1}^{\operatorname{tr}}$ is close to or equal to zero, then the posterior density of $\pm \sqrt{\theta_{1}}$ is likely to be centered around zero.

For illustration, we consider posterior inference for $T=100$ observations simulated from the local level model (21) with $\sigma_{\varepsilon}^{2}=0.01$ and two different values for $\theta_{1}$, namely $\theta_{1}^{\operatorname{tr}}=0.01$ and $\theta_{1}^{\operatorname{tr}}=0$. For any type of prior distribution, the posterior distribution of $\pm \sqrt{\theta_{1}}$ is derived using numerical integration. For both values of $\theta_{1}^{\operatorname{tr}}$, the posterior of $\pm \sqrt{\theta_{1}}$ is plotted in Figure 2 under the inverted Gamma prior $\theta_{1} \sim \mathcal{G}^{-1}\left(0.5, D_{0}\right)$ as well as under the normal prior $\pm \sqrt{\theta_{1}} \sim \mathcal{N}\left(0, B_{0} \sigma_{\varepsilon}^{2}\right)$ for various scale parameters $D_{0}$ and $B_{0}$.

Whereas the posterior is fairly robust to the choice of the hyperparameter $B_{0}$ in the normal prior, it turns out to be rather sensitive to the hyperparameter $D_{0}$ of the inverted Gamma prior. Both posteriors are roughly the same for $\theta_{1}^{\text {tr }}=0.01$ and clearly indicate that $\theta_{1}^{\operatorname{tr}}>0$. A remarkable difference, however, occurs if $\theta_{1}^{\operatorname{tr}}=0$. Under the normal prior, 
the posterior of $\pm \sqrt{\theta_{1}}$ is centered at 0 strongly supporting the hypothesis that $\theta_{1}^{\operatorname{tr}}=0$. The inverted Gamma density, however, shrinks the posterior of $\pm \sqrt{\theta_{1}}$ away from 0 , falsely indicating that $\theta_{1}^{\operatorname{tr}}>0$.

\subsection{MCMC Estimation}

An MCMC approach is implemented to sample jointly the indicators $(\boldsymbol{\delta}, \boldsymbol{\gamma})=\left(\delta, \gamma_{1}, \gamma_{2}\right)$, the unrestricted elements of the parameter $\boldsymbol{\beta}=\left(\mu_{0}, a_{0}, \sqrt{\theta_{1}}, \sqrt{\theta_{2}}\right)$, the observation variance $\sigma_{\varepsilon}^{2}$, and the latent state process $\mathbf{x}=\left(\mathbf{x}_{1}, \ldots, \mathbf{x}_{T}\right)$, where $\mathbf{x}_{t}$ is the state vector defined in (9).

When sampling the indicators $(\boldsymbol{\delta}, \boldsymbol{\gamma})$ we marginalize over the parameters for which variable selection is carried out, as suggested by Geweke (1996) and Smith and Kohn (1996), see also George and McCulloch (1997). To make this feasible, we use the noncentered parameterization of the dynamic linear trend model. Conditional on the state process $\mathbf{x}=\left(\mathbf{x}_{1}, \ldots, \mathbf{x}_{T}\right)$, the observation equation (14) defines a standard regression model

$$
y_{t}=\mathbf{z}_{t}^{\boldsymbol{\delta}, \boldsymbol{\gamma}} \boldsymbol{\beta}^{\boldsymbol{\delta}, \boldsymbol{\gamma}}+\varepsilon_{t}, \quad \varepsilon_{t} \sim \mathcal{N}\left(0, \sigma_{\varepsilon}^{2}\right) .
$$

If all indicators take the value one, then $\boldsymbol{\beta}^{\boldsymbol{\delta}, \boldsymbol{\gamma}}=\boldsymbol{\beta}$ and $\mathbf{z}_{t}^{\boldsymbol{\delta}, \boldsymbol{\gamma}}=\mathbf{z}_{t}$, where $\mathbf{z}_{t}=\left(1, t, \tilde{\mu}_{t}, \tilde{A}_{t}\right)$. Otherwise the restricted parameter $\boldsymbol{\beta}^{\boldsymbol{\delta}, \boldsymbol{\gamma}}$ and the corresponding predictors $\mathbf{z}_{t}^{\boldsymbol{\delta}, \boldsymbol{\gamma}}$ contain only those elements of $\boldsymbol{\beta}$ and $\mathbf{z}_{t}$, respectively, for which the corresponding indicator is equal to 1 . Under the conjugate prior

$$
\boldsymbol{\beta}^{\boldsymbol{\delta}, \boldsymbol{\gamma}} \sim \mathcal{N}\left(\mathbf{a}_{0}^{\boldsymbol{\delta}, \boldsymbol{\gamma}}, \mathbf{A}_{0}^{\boldsymbol{\delta}, \boldsymbol{\gamma}} \sigma_{\varepsilon}^{2}\right), \quad \sigma_{\varepsilon}^{2} \sim \mathcal{G}^{-1}\left(c_{0}, C_{0}\right)
$$

the posterior $p(\boldsymbol{\delta}, \boldsymbol{\gamma} \mid \mathbf{x}, \mathbf{y})$ is obtained from Bayes' theorem:

$$
p(\boldsymbol{\delta}, \boldsymbol{\gamma} \mid \mathbf{x}, \mathbf{y}) \propto p(\mathbf{y} \mid \boldsymbol{\delta}, \boldsymbol{\gamma}, \mathbf{x}) p(\boldsymbol{\delta}, \boldsymbol{\gamma})
$$

where $p(\mathbf{y} \mid \boldsymbol{\delta}, \boldsymbol{\gamma}, \mathbf{x})$ is equal to the marginal likelihood of the regression model (22):

$$
p(\mathbf{y} \mid \boldsymbol{\delta}, \boldsymbol{\gamma}, \mathbf{x})=\frac{1}{(2 \pi)^{T / 2}} \frac{\left|\mathbf{A}_{T}^{\boldsymbol{\delta}, \boldsymbol{\gamma}}\right|^{1 / 2}}{\left|\mathbf{A}_{0}^{\boldsymbol{\delta}, \boldsymbol{\gamma}}\right|^{1 / 2}} \frac{\Gamma\left(c_{T}\right) C_{0}^{c_{0}}}{\Gamma\left(c_{0}\right)\left(C_{T}^{\boldsymbol{\delta}, \boldsymbol{\gamma}}\right)^{c_{T}}} .
$$

Here $\mathbf{A}_{T}^{\boldsymbol{\delta}, \boldsymbol{\gamma}}, c_{T}$ and $C_{T}^{\boldsymbol{\delta}, \boldsymbol{\gamma}}$ denote the posterior moments of $\boldsymbol{\beta}^{\boldsymbol{\delta}, \boldsymbol{\gamma}}$ and $\sigma_{\varepsilon}^{2}$ given below in (26) to (28). It should be noted that such a closed form expression for $p(\mathbf{y} \mid \boldsymbol{\delta}, \boldsymbol{\gamma}, \mathbf{x})$ is not 
available if any of the indicators $\gamma_{1}$ and $\gamma_{2}$ is equal to 1 and an inverted Gamma prior is chosen for $\theta_{1}$ and $\theta_{2}$. The MCMC scheme reads:

(a) Sample the indicators $(\boldsymbol{\delta}, \gamma)=\left(\delta, \gamma_{1}, \gamma_{2}\right)$, the initial values $\mu_{0}$ and $a_{0}$, all variance parameters $\sqrt{\theta_{1}}$ and $\sqrt{\theta_{2}}$ and the observation variance $\sigma_{\varepsilon}^{2}$ jointly in one block:

(a1) Sample the indicators from $p(\boldsymbol{\delta}, \boldsymbol{\gamma} \mid \mathbf{x}, \mathbf{y})$ given in (24);

(a2) sample $\sigma_{\varepsilon}^{2}$ from $\mathcal{G}^{-1}\left(c_{T}, C_{T}^{\boldsymbol{\delta}, \boldsymbol{\gamma}}\right)$, and, conditional on $\sigma_{\varepsilon}^{2}$, sample $\mu_{0}, a_{0}$ (if unrestricted), and all unrestricted variance parameters $\sqrt{\theta_{1}}$ and $\sqrt{\theta_{2}}$ jointly from the normal posterior $\mathcal{N}\left(\mathbf{a}_{T}^{\boldsymbol{\delta}, \boldsymbol{\gamma}}, \mathbf{A}_{T}^{\boldsymbol{\delta}, \boldsymbol{\gamma}} \sigma_{\varepsilon}^{2}\right)$ where

$$
\begin{aligned}
& \mathbf{A}_{T}^{\boldsymbol{\delta}, \boldsymbol{\gamma}}=\left(\left(\mathbf{Z}^{\boldsymbol{\delta}, \boldsymbol{\gamma}}\right)^{\prime} \mathbf{Z}^{\boldsymbol{\delta}, \boldsymbol{\gamma}}+\left(\mathbf{A}_{0}^{\boldsymbol{\delta}, \boldsymbol{\gamma}}\right)^{-1}\right)^{-1}, \\
& \mathbf{a}_{T}^{\boldsymbol{\delta}, \boldsymbol{\gamma}}=\mathbf{A}_{T}^{\boldsymbol{\delta}, \boldsymbol{\gamma}}\left(\left(\mathbf{Z}^{\boldsymbol{\delta}, \boldsymbol{\gamma}}\right)^{\prime} \mathbf{y}+\left(\mathbf{A}_{0}^{\boldsymbol{\delta}, \boldsymbol{\gamma}}\right)^{-1} \mathbf{a}_{0}^{\boldsymbol{\delta}, \boldsymbol{\gamma}}\right), \\
& c_{T}=c_{0}+T / 2, \\
& C_{T}^{\boldsymbol{\delta}, \boldsymbol{\gamma}}=C_{0}+\frac{1}{2}\left(\mathbf{y}^{\prime} \mathbf{y}+\left(\mathbf{a}_{0}^{\boldsymbol{\delta}, \boldsymbol{\gamma}}\right)^{\prime}\left(\mathbf{A}_{0}^{\boldsymbol{\delta}, \boldsymbol{\gamma}}\right)^{-1} \mathbf{a}_{0}^{\boldsymbol{\delta}, \boldsymbol{\gamma}}-\left(\mathbf{a}_{T}^{\boldsymbol{\delta}, \boldsymbol{\gamma}}\right)^{\prime}\left(\mathbf{A}_{T}^{\boldsymbol{\delta}, \boldsymbol{\gamma}}\right)^{-1} \mathbf{a}_{T}^{\boldsymbol{\delta}, \boldsymbol{\gamma}}\right),
\end{aligned}
$$

and $\mathbf{Z}^{\boldsymbol{\delta}, \boldsymbol{\gamma}}$ is the regressor matrix with rows equal to $\mathbf{z}_{t}^{\boldsymbol{\delta}, \boldsymbol{\gamma}}$;

(a3) set all restricted initial values and all restricted variances equal to 0.

(b) Sample $\mathbf{x}=\left(\mathbf{x}_{1}, \ldots, \mathbf{x}_{T}\right)$ from the state space form (20).

(c) Perform a random sign switch for $\sqrt{\theta_{1}}$ and $\left\{\tilde{\mu}_{t}\right\}_{1}^{T}$. Thus with probability 0.5 the draws of these parameters remain unchanged, while they are substituted by $-\sqrt{\theta_{1}}$ and $\left\{-\tilde{\mu}_{t}\right\}_{1}^{T}$ with the same probability. Perform another random sign switch for $\sqrt{\theta_{2}},\left\{\tilde{a}_{t}\right\}_{1}^{T}$ and $\left\{\tilde{A}_{t}\right\}_{1}^{T}$.

A few comments are in order. The dimension of the normal distribution appearing in step (a2) depends on the number of unrestricted components and is equal to $1+\delta+\gamma_{1}+\gamma_{2}$.

In step (b), forward-filtering-backward-sampling (FFBS, Frühwirth-Schnatter (1994); Carter and Kohn (1994); De Jong and Shephard (1995)) is used to sample $\mathbf{x}=\left(\mathbf{x}_{1}, \ldots, \mathbf{x}_{T}\right)$. To speed up sampling, a reduced state space form is used if $\gamma_{1}$ or $\gamma_{2}$ is 0 . If, for instance, $\gamma_{1}=0$, then the observation equation is independent of $\left\{\tilde{\mu}_{t}\right\}_{1}^{T}$. FFBS is applied to the reduced state vector $\mathbf{x}_{t}=\left(\tilde{a}_{t}, \tilde{A}_{t}\right)^{\prime}$, while $\tilde{\mu}_{1}, \ldots, \tilde{\mu}_{T}$ is sampled from (11). A similar method 
applies, if $\gamma_{2}=0$, with reduced state vector $x_{t}=\tilde{\mu}_{t}$. If both indicators $\gamma_{1}$ and $\gamma_{2}$ are equal to 0, then no FFBS is needed, as sampling of $\mathbf{x}$ from the prior is straightforward.

Sampling of the state process in step (b) is based on the noncentered parameterization. The unknown components $a_{t}$ and $\mu_{t}$ in the centered parameterization are easily reconstructed from the MCMC draws using (15) and (16).

We found it useful to start from an unrestricted model and to run the first say 1000 draws of burn-in without variable selection. This allows to generate sensible starting values for the state process and the parameters of the unrestricted model before variable selection actually sets in.

\section{Extension to the Basic Structural Model}

\subsection{The Parsimonious Basic Structural Model}

In the basic structural model, a seasonal component is added to the dynamic linear trend model discussed in Section 2, see e.g. Harvey (1989):

$$
\begin{aligned}
& s_{t}=-s_{t-1}-\cdots-s_{t-S+1}+\omega_{3 t}, \quad \omega_{3 t} \sim \mathcal{N}\left(0, \theta_{3}\right), \\
& y_{t}=\mu_{t}+s_{t}+\varepsilon_{t}, \quad \varepsilon_{t} \sim \mathcal{N}\left(0, \sigma_{\varepsilon}^{2}\right),
\end{aligned}
$$

where $\mu_{t}$ is the same as in (2) and (3) and $S$ is the number of seasons. The initial seasonal pattern is given by $\mathbf{s}_{0}=\left(s_{-S+1}, \ldots, s_{0}\right)$ with $s_{-S+1}+\ldots+s_{0}=0$. In addition to the model specification problems discussed in Section 2, a decision has to be made if a seasonal pattern is present and if this pattern is fixed or dynamic. To this aim, two additional binary stochastic indicators $\delta_{3}$ and $\gamma_{3}$ are introduced. $\delta_{3}$ decides, if the initial seasonal pattern is equal to 0 , whereas $\gamma_{3}$ controls if it changes over time. As before, the indicators are introduced into the noncentered version of the model.

Combine the following stochastic difference equation:

$$
\tilde{s}_{t}=-\tilde{s}_{t-1}-\cdots-\tilde{s}_{t-S+1}+\tilde{\omega}_{3 t}, \quad \tilde{\omega}_{3 t} \sim \mathcal{N}(0,1),
$$

where $\tilde{s}_{-S+1}=\ldots=\tilde{s}_{0}=0$ with the state equations (6) to $(7)$ and following observation equation:

$$
y_{t}=\mu_{t}+\delta_{3} s_{0, q(t)}+\gamma_{3} \sqrt{\theta_{3}} \tilde{s}_{t}+\varepsilon_{t}, \quad \varepsilon_{t} \sim \mathcal{N}\left(0, \sigma_{\varepsilon}^{2}\right)
$$


where $\theta_{3}$ is equal to the variance of the error term in (29), $\mu_{t}$ is the same as in (16) and $s_{0, q(t)}$ with $q(t)=1+(t-1) \bmod S$ is the seasonal component corresponding to time $t$. The resulting state space model is a noncentered parameterization of the basic structural model.

If $\gamma_{3}=0$, we define $\theta_{3}=0$ and the resulting seasonal pattern is fixed. If $\delta_{3}=0$, we set the initial seasonal pattern to zero, $\mathbf{s}_{0}=\mathbf{0}$. If both indicators are equal to 0 , then no seasonal pattern is present in the time series and the model reduces to the dynamic linear trend model studied in Section 2.

The non-centered parameterization (32) could be written as

$$
y_{t}=\mu_{0}+\delta t a_{0}+\delta_{3} s_{0, q(t)}+\gamma_{1} \sum_{j=1}^{t} \omega_{1 j}+\gamma_{2} \sum_{j=1}^{t-1}(t-j) \omega_{2 j}+\gamma_{3} \sum_{j=1}^{t} \omega_{3 j}+\varepsilon_{t},
$$

with fixed effects $\mu_{0}, a_{0}$ and $s_{0, q(t)}$ and random effects $\omega_{1 j}, \omega_{2 j}$ and $\omega_{3 j}$. Evidently, all $2^{5}=32$ combinations of indicators are identifiable.

As before, the noncentered model is not identified, as the sign of $\sqrt{\theta_{3}}$ and the sequence $\left\{\tilde{s}_{t}\right\}_{1}^{T}$ may be changed without changing the likelihood function. As a consequence, the likelihood function $p(\mathbf{y} \mid \boldsymbol{\vartheta})$ where $\boldsymbol{\vartheta}=\left(\sqrt{\theta_{1}}, \sqrt{\theta_{2}}, \sqrt{\theta_{3}}, \mu_{0}, a_{0}, \mathbf{s}_{0}, \sigma_{\varepsilon}^{2}\right)$ is symmetric around 0 in the direction of $\sqrt{\theta_{i}}, i=1,2,3$. With an increasing number of observations $T$, the modes of the likelihood function will be close to all combinations of $\left( \pm \sqrt{\theta_{1}^{\operatorname{tr}}}, \pm \sqrt{\theta_{2}^{\operatorname{tr}}}, \pm \sqrt{\theta_{3}^{\operatorname{tr}}}, \boldsymbol{\xi}^{\operatorname{tr}}\right)$, where $\boldsymbol{\xi}^{\mathrm{tr}}=\left(\mu_{0}^{\mathrm{tr}}, a_{0}^{\mathrm{tr}}, \mathbf{s}_{0}^{\mathrm{tr}}, \sigma_{\varepsilon}^{2, \mathrm{tr}}\right)$. Thus with an increasing number of observations, the likelihood function has eight modes as long as in the data generating process the true variances $\theta_{1}^{\operatorname{tr}}, \theta_{2}^{\operatorname{tr}}$ and $\theta_{3}^{\operatorname{tr}}$ are positive. If one of the true variances is equal to 0 while the others are positive, half of those modes are identical leaving four modes. If two of the true variances are equal to 0 while the other is positive, only two modes are different leaving a bimodal likelihood with an increasing number of observations $T$. If all variances are equal to zero, then the likelihood function will be unimodal with an increasing number of observations $T$.

It is easy to verify that in the centered parameterization the parsimonious model is equivalent to combining (2) and (3) with state equation (29) and following observation equation:

$$
y_{t}=\mu_{t}+\delta_{3} s_{0, q(t)}+\gamma_{3}\left(s_{t}-\delta_{3} s_{0, q(t)}\right)+\varepsilon_{t}, \quad \varepsilon_{t} \sim \mathcal{N}\left(0, \sigma_{\varepsilon}^{2}\right)
$$




\subsection{MCMC Sampling Scheme}

An MCMC approach is implemented to sample the indicators $\boldsymbol{\delta}=\left(\delta, \delta_{3}\right)$ and $\boldsymbol{\gamma}=$ $\left(\gamma_{1}, \gamma_{2}, \gamma_{3}\right)$, the model parameters $\boldsymbol{\beta}=\left(\mu_{0}, a_{0}, \mathbf{s}_{0}, \sqrt{\theta_{1}}, \sqrt{\theta_{2}}, \sqrt{\theta_{3}}\right)$, the observation variance $\sigma_{\varepsilon}^{2}$, and the latent state process $\mathbf{x}=\left(\mathbf{x}_{1}, \ldots, \mathbf{x}_{T}\right)$, where $\mathbf{x}_{t}$ is following state vector:

$$
\mathbf{x}_{t}=\left(\begin{array}{llllll}
\tilde{\mu}_{t} & \tilde{a}_{t} & \tilde{A}_{t} & \tilde{s}_{t} & \ldots & \tilde{s}_{t-S+2}
\end{array}\right)^{\prime} .
$$

The MCMC sampling scheme introduced in Subsection 2.4 is easily modified to deal with a basic structural model. Conditional on the state process $\mathbf{x}=\left(\mathbf{x}_{1}, \ldots, \mathbf{x}_{T}\right)$, the observation equation (32) of the non-centered parameterization of the basic structural model is a standard regression model as in (22) with appropriate regressors $\mathbf{z}_{t}^{\boldsymbol{\delta}, \boldsymbol{\gamma}}$. Under the same conditionally conjugate prior for $\boldsymbol{\beta}^{\boldsymbol{\delta}, \boldsymbol{\gamma}}$ and $\sigma_{\varepsilon}^{2}$ as in (23), the marginal likelihood $p(\mathbf{y} \mid \boldsymbol{\delta}, \boldsymbol{\gamma}, \mathbf{x})$ and all posterior moments are then computed exactly as in Subsection 2.4. This leads to following MCMC scheme:

(a) Sample the indicators $(\boldsymbol{\delta}, \boldsymbol{\gamma})$, the observation variance $\sigma_{\varepsilon}^{2}$ and the initial values $\mu_{0}$, $a_{0}$, and $\mathbf{s}_{0}$ and all variance parameters $\sqrt{\theta_{1}}, \sqrt{\theta_{2}}$ and $\sqrt{\theta_{3}}$ jointly in one block as in Subsection 2.4.

(b) Sample $\mathbf{x}=\left(\mathbf{x}_{1}, \ldots, \mathbf{x}_{T}\right)$ from the state space form corresponding to (32).

(c) Perform two random sign switches as in step (c) in Subsection 2.4. Perform a third random sign switch for $\sqrt{\theta_{3}}$ and $\left\{\tilde{s}_{t}\right\}_{1}^{T}$.

As in Subsection 2.4, FFBS is applied to a reduced state vector, if any of the indicators $\gamma_{i}=0$ is equal to 0 , while the remaining components are sampled from the prior.

\subsection{Prior Specification}

To run the MCMC schemes, prior distributions have to be defined. As before, we assume a uniform prior distribution over all possible indicators $\boldsymbol{\delta}$ and $\boldsymbol{\gamma}$.

For the observation variance $\sigma_{\varepsilon}^{2}$ we choose a hierarchical prior where $\sigma_{\varepsilon}^{2} \sim \mathcal{G}^{-1}\left(c_{0}, C_{0}\right)$ and $C_{0} \sim \mathcal{G}\left(g_{0}, G_{0}\right)$ with $c_{0}=2.5, g_{0}=5$ and $G_{0}=g_{0} /\left(0.75 \operatorname{Var}(y)\left(c_{0}-1\right)\right)$. For this hierarchical prior it is necessary to add an additional sampling step were $C_{0}$ is sampled 
conditional on $\sigma_{\varepsilon}^{2}$ from the conditional Gamma posterior $C_{0} \mid \sigma_{\varepsilon}^{2} \sim \mathcal{G}\left(g_{0}+c_{0}, G_{0}+1 / \sigma_{\varepsilon}^{2}\right)$ at each sweep of the sampler.

Prior (23) assumes normality not only for the initial values $\mu_{0}, a_{0}$, and $\mathbf{s}_{0}$, but also for all remaining parameters. For the same reasons as in Subsection 2.3, we do not use inverted Gamma priors for the variances $\theta_{1}, \ldots, \theta_{3}$ as usual in the basic structural model, but assume that the parameters $\pm \sqrt{\theta_{1}}, \pm \sqrt{\theta_{2}}$ and $\pm \sqrt{\theta_{3}}$ follow a normal prior.

In our case studies, we found the following prior choices useful for variable selection. First, we use a partially proper prior which combines the improper prior $p\left(\mu_{0}\right) \propto 1$ for $\mu_{0}$ with a proper prior $\mathcal{N}\left(\mathbf{0}, \mathbf{B}_{0}^{\boldsymbol{\delta}, \boldsymbol{\gamma}} \sigma_{\varepsilon}^{2}\right)$ on the remaining unrestricted elements of $\boldsymbol{\beta}^{\boldsymbol{\delta}, \boldsymbol{\gamma}}$, where $\mathbf{B}_{0}^{\boldsymbol{\delta}, \boldsymbol{\gamma}}=B_{0} \mathbf{I}$. This prior corresponds to choosing $\mathbf{a}_{0}^{\boldsymbol{\delta}, \boldsymbol{\gamma}}=\mathbf{0}$ and

$$
\left(\mathbf{A}_{0}^{\boldsymbol{\delta}, \boldsymbol{\gamma}}\right)^{-1}=\left(\begin{array}{ll}
0 & \\
& \left(\mathbf{B}_{0}^{\boldsymbol{\delta}, \boldsymbol{\gamma}}\right)^{-1}
\end{array}\right) .
$$

Under this prior, the sampling scheme described above has to be changed slightly, because the marginal likelihood $p(\mathbf{y} \mid \boldsymbol{\delta}, \boldsymbol{\gamma}, \mathbf{x})$ and the posterior parameter $c_{T}$ read:

$$
\begin{aligned}
& p(\mathbf{y} \mid \boldsymbol{\delta}, \boldsymbol{\gamma}, \mathbf{x})=\frac{1}{(2 \pi)^{(T-1) / 2}} \frac{\left|\mathbf{A}_{T}^{\boldsymbol{\delta}, \boldsymbol{\gamma}}\right|^{1 / 2}}{\left|\mathbf{B}_{0}^{\boldsymbol{\delta}, \boldsymbol{\gamma}}\right|^{1 / 2}} \frac{\Gamma\left(c_{T}\right) C_{0}^{c_{0}}}{\Gamma\left(c_{0}\right)\left(C_{T}^{\boldsymbol{\delta}, \boldsymbol{\gamma}}\right)^{c_{T}}}, \\
& c_{T}=c_{0}+(T-1) / 2 .
\end{aligned}
$$

Another prior commonly used in model selection is the fractional prior (O'Hagan, 1995). In the present context, this is a conditional fractional prior for regression model (22) which depends on the state vector $\mathbf{x}$ and is defined as

$$
p\left(\boldsymbol{\beta}^{\boldsymbol{\delta}, \boldsymbol{\gamma}} \mid \sigma_{\varepsilon}^{2}\right) \propto p\left(\mathbf{y} \mid \boldsymbol{\beta}^{\boldsymbol{\delta}, \boldsymbol{\gamma}}, \sigma_{\varepsilon}^{2}\right)^{b}=\left(\frac{1}{2 \pi \sigma_{\varepsilon}^{2}}\right)^{T b / 2} \exp \left(-\frac{b}{2 \sigma_{\varepsilon}^{2}}\left(\mathbf{y}-\mathbf{Z}^{\boldsymbol{\delta}, \boldsymbol{\gamma}} \boldsymbol{\beta}^{\boldsymbol{\delta}, \boldsymbol{\gamma}}\right)^{\prime}\left(\mathbf{y}-\mathbf{Z}^{\boldsymbol{\delta}, \boldsymbol{\gamma}} \boldsymbol{\beta}^{\boldsymbol{\delta}, \boldsymbol{\gamma}}\right)\right) .
$$

The fractional prior can be interpreted as posterior of a non-informative prior and a fraction $b$ of the data $\mathbf{y}$. It reads

$$
\boldsymbol{\beta}^{\boldsymbol{\delta}, \boldsymbol{\gamma}} \mid \sigma_{\varepsilon}^{2} \sim \mathcal{N}\left(\mathbf{a}_{T}^{\boldsymbol{\delta}, \boldsymbol{\gamma}}, \mathbf{A}_{T}^{\boldsymbol{\delta}, \boldsymbol{\gamma}} \sigma_{\varepsilon}^{2} / b\right)
$$

where $\mathbf{a}_{T}^{\boldsymbol{\delta}, \boldsymbol{\gamma}}$ and $\mathbf{A}_{T}^{\boldsymbol{\delta}, \boldsymbol{\gamma}}$ are the posterior moments under a non-informative prior:

$$
\mathbf{A}_{T}^{\boldsymbol{\delta}, \boldsymbol{\gamma}}=\left(\left(\mathbf{Z}^{\boldsymbol{\delta}, \boldsymbol{\gamma}}\right)^{\prime} \mathbf{Z}^{\boldsymbol{\delta}, \boldsymbol{\gamma}}\right)^{-1}, \quad \mathbf{a}_{T}^{\boldsymbol{\delta}, \boldsymbol{\gamma}}=\mathbf{A}_{T}^{\boldsymbol{\delta}, \boldsymbol{\gamma}}\left(\mathbf{Z}^{\boldsymbol{\delta}, \boldsymbol{\gamma}}\right)^{\prime} \mathbf{y}
$$


In the MCMC sampling scheme all posterior moments as well as the marginal likelihood $p(\mathbf{y} \mid \boldsymbol{\delta}, \boldsymbol{\gamma}, \mathbf{x})$ have to be modified according to:

$$
\begin{aligned}
& c_{T}=c_{0}+\frac{(1-b)}{2} T, \quad C_{T}^{\boldsymbol{\delta}, \boldsymbol{\gamma}}=C_{0}+\frac{(1-b)}{2}\left(\mathbf{y}^{\prime} \mathbf{y}-\left(\mathbf{a}_{T}^{\boldsymbol{\delta}, \boldsymbol{\gamma}}\right)^{\prime}\left(\mathbf{A}_{T}^{\boldsymbol{\delta}, \boldsymbol{\gamma}}\right)^{-1} \mathbf{a}_{T}^{\boldsymbol{\delta}, \boldsymbol{\gamma}}\right), \\
& p(\mathbf{y} \mid \boldsymbol{\delta}, \boldsymbol{\gamma}, \mathbf{x})=\frac{b^{q / 2} \Gamma\left(c_{T}\right) C_{0}^{c_{0}}}{(2 \pi)^{T(1-b) / 2} \Gamma\left(c_{0}\right)\left(C_{T}^{\boldsymbol{\delta}, \boldsymbol{\gamma}}\right)^{c_{T}}}
\end{aligned}
$$

where $q$ is the dimension of $\boldsymbol{\beta}^{\boldsymbol{\delta}, \boldsymbol{\gamma}}$, while $\mathbf{a}_{T}^{\boldsymbol{\delta}, \boldsymbol{\gamma}}$ and $\mathbf{A}_{T}^{\boldsymbol{\delta}, \boldsymbol{\gamma}}$ are the same as in (35).

\subsection{UK coal consumption data}

We reconsider the series of UK coal consumption, analyzed in Harvey (1989), FrühwirthSchnatter (1994) and Frühwirth-Schnatter (1995), among others. Data are quarterly from 1/1960 to 4/1982, see Figure 3, panel (a). We model the series on the log scale by a basic structural model.

All subsequent implementation are carried out using MATLAB (Version 7.2.0) on a notebook with a $2.0 \mathrm{GHz}$ processor.

\subsubsection{Bayesian Inference for the Unrestricted Basic Structural Model}

For illustration, we start with inference for the unrestricted basic structural model without variable selection and compare the priors $\theta_{i} \sim \mathcal{G}^{-1}\left(-0.5,10^{-7}\right)$ with the priors $\pm \sqrt{\theta_{i}} \sim$ $\mathcal{N}(0,1)$. The remaining priors are $\mu_{0} \sim \mathcal{N}\left(0,100 \sigma_{\varepsilon}^{2}\right)$ and $\sigma_{\varepsilon}^{2} \sim \mathcal{G}^{-1}(0,0)$.

Estimated state components are plotted for the inverted Gamma prior in Figure 3. The posterior densities of the transformed process variances $\pm \sqrt{\theta_{i}}, i=1, \ldots, 3$ are plotted in Figure 4. Evidently, the posterior density of any parameter $\pm \sqrt{\theta_{i}}$ has to be symmetric around zero. If the unknown variance $\theta_{i}$ is systematically different from zero, then the posterior density of $\pm \sqrt{\theta_{i}}$ is likely to be bimodal; otherwise, if $\theta_{i}$ is close to zero, the posterior density of $\pm \sqrt{\theta_{i}}$ will be centered around zero. This should allow to explore the hypothesis that $\theta_{i}=0$.

For the normal prior $\pm \sqrt{\theta_{i}} \sim \mathcal{N}(0,1)$, the posterior densities of $\pm \sqrt{\theta_{1}}$ and $\pm \sqrt{\theta_{3}}$ are unimodal and centered at 0 , while the posterior of $\pm \sqrt{\theta_{2}}$ is bimodal. This indicates 
that $\theta_{1}$ and $\theta_{3}$ are equal to 0 , while $\theta_{2}>0$. This finding is confirmed by stochastic model selection search in Subsection 3.4.2. Under the inverted Gamma prior, all posterior densities are bimodal and $\pm \sqrt{\theta_{i}}$ is bounded away from 0 , providing spurious evidence for an unrestricted model.

The MCMC draws underlying Figure 4 were obtaining by running MCMC sampling for 40,000 iterations after a burn-in of 10,000. For the normal prior we use the new MCMC scheme presented in Subsection 3.2 which is based on the non-centered parameterization of the basic structural model. For the inverted Gamma prior we use the usual two-step Gibbs sampler which is based on the centered parameterization, see e.g. Durbin and Koopman (2001). MCMC draws for $\pm \sqrt{\theta_{i}}$ are obtained by multiplying the square root of the MCMC draws $\theta_{i}^{(m)}$ with a random sign.

An interesting difference between the centered and the non-centered parameterization lies in the mixing properties of the corresponding MCMC draws. If some variances are equal to or close to 0, the corresponding MCMC draws mix badly under the centered parameterizaton, while mixing is perfect under the noncentered parameterizaton, see Figure 5.

\subsubsection{Stochastic Model Specification Search}

Stochastic model specification search is carried out using the prior described in Subsection 3.3. We compare partially proper priors with different prior variances $B_{0}$ with fractional priors with different fractions $b$. For each prior, MCMC sampling was carried out for $M=100,000$ draws after a burn-in of 20,000 draws. The first 1000 draws of the burn-in were drawn from the unrestricted model, model selection began after these first 1000 draws. Depending on the prior, running MCMC takes between 43 and 52 minutes, see also Table 2.

Results of the variable selection procedure are summarized in Table 1 and 2. The most frequently visited model in Table 1 is robust against the prior choice, only the frequency with which this model is selected varies. The same model results for all priors, if in Table 2 an indicator is estimated to be 1 , if the corresponding posterior inclusion probability is greater than or equal to 0.5 . 
As expected from panel (a) and (d) in Figure 3, a seasonal pattern is present in the selected model $\left(\delta_{3}=1\right)$, but it is fixed and does not change over time $\left(\gamma_{3}=0\right)$. The drift $a_{t}$ is stochastic $\left(\gamma_{2}=1\right)$, but the initial value $a_{0}$ is selected to be $0(\delta=0)$. This is plausible from panel (c) in Figure 3, where the pointwise confidence band covers $a_{t}=0$ at $t=0$, but does not contain the restricted line where $a_{t}=0$ for all $t$. Finally, no additional noise $\omega_{1 t}$ is added in (2), since $\gamma_{1}=0$.

\subsubsection{Comparison to Marginal Likelihood Computation}

For this case study, it is possible to compare variable selection to the more traditional approach of computing marginal likelihoods $p(\mathbf{y} \mid \mathcal{M})$ of all 32 models $\mathcal{M}=\left(\delta, \delta_{3}, \gamma_{1}, \gamma_{2}, \gamma_{3}\right)$, obtained by all possible combinations of indicators. Because we are dealing with a linear Gaussian state space model it is possible to integrate out all states and the initial values by running a Kalman filter and we are left with a low dimensional integration over the parameter $\left(\boldsymbol{\theta}^{\mathcal{M}}, \sigma_{\varepsilon}^{2}\right)$ where $\boldsymbol{\theta}^{\mathcal{M}}$ contains all unconstrained variances $\theta_{1}, \theta_{2}$ and $\theta_{3}$ present in model $\mathcal{M}$.

Each marginal likelihood $p(\mathbf{y} \mid \mathcal{M})$ is computed by importance sampling with 20000 draws from the following importance density. For each model, MCMC for the noncentered parameterization with fixed indicators $(\boldsymbol{\delta}, \boldsymbol{\gamma})$ was run for 40, 000 iterations after a burn-in of 10,000 draws. The density of a $t_{\nu}(\boldsymbol{m}, \boldsymbol{S})$-distribution with $\nu=10$ was fitted to the MCMC draws of $\left(\log \left(\boldsymbol{\theta}^{\mathcal{M}}\right), \log \left(\sigma_{\varepsilon}^{2}\right)\right)$ by matching moments, i.e. $\boldsymbol{m}$ is equal to the mean of the transformed MCMC draws, while $\boldsymbol{S}$ is equal to the covariance matrix times $(\nu-2) / \nu$. Computation time for a single model lies between 15 and $16 \mathrm{CPU}$ minutes. Computation for all 32 models took 494 CPU minutes.

The resulting estimators, together with their standard errors, are reported in Table 3 and indicate that importance sampling yields very precise estimates of the marginal likelihood.

Marginal likelihood computations are based on following priors. We choose the same hierarchical prior on the observation variance $\sigma_{\varepsilon}^{2}$ as in Subsection 3.4.2 and marginalize over the random hyperparameter $C_{0}$ to facilitate computation of the marginal likelihood:

$$
p\left(\sigma_{\varepsilon}^{2} \mid c_{0}, g_{0}, G_{0}\right)=\frac{G_{0}^{g_{0}} \Gamma\left(c_{0}+g_{0}\right)}{\Gamma\left(c_{0}\right) \Gamma\left(g_{0}\right)\left(\sigma_{\varepsilon}^{2}\right)^{c_{0}+1}}\left(G_{0}+1 / \sigma_{\varepsilon}^{2}\right)^{-\left(c_{0}+g_{0}\right)} .
$$


Since marginal likelihood computation is not possible for improper priors, we modify the partially proper used in Subsection 3.4.2 for variable selection slightly by assuming $\mu_{0} \sim \mathcal{N}\left(0,10000 \sigma_{\varepsilon}^{2}\right)$, whereas we use the same $\mathcal{N}\left(0, B_{0} \sigma_{\varepsilon}^{2}\right)$ prior with $B_{0}=1$ and $B_{0}=$ 100 , respectively, for the initial value of all other components and the process standard deviations. We did not consider the fractional prior, because it is defined as a conditional prior given the all states, which makes it impossible to integrate out the high-dimensional state vector in the fashion described above.

The same prior is used for the stochastic model specification search algorithm which was run for 100,000 iterations after a burn-in of 20000. Computation time for running variable selection is about $52 \mathrm{CPU}$ minutes which is considerably faster than computing the marginal likelihood for all models.

Table 3 reports the number $h(\mathcal{M})$ of times the model $\mathcal{M}$ was visited during the 100,000 MCMC iterations. We find that the relative frequency $h(\mathcal{M}) / 100,000$ is close the model probability $\hat{p}(\mathcal{M} \mid \mathbf{y})$ which is estimated from the marginal likelihood $\hat{p}(\mathbf{y} \mid \mathcal{M})$ under the uniform prior $p(\mathcal{M})=1 / 32$ underlying variable selection.

In Table 1 we found that changing the prior had no effect on the best model, however, the ranking of the remaining models changed. From Table 3 we see that this effect is also present for the marginal likelihood. This sensitivity of the marginal likelihood to prior choice was even more pronounced when we considered the conventional inverted Gamma prior for the process variances $\theta_{i}$.

\section{Model Selection for Non-Gaussian State Space Mod- els}

The investigations in Shively et al. (1999) show that variable selection in state space models is also feasible for binary data. In this section we show how the variable selection approach developed in Section 2 and Section 3 for Gaussian state space model may be extended to nonnormal state space models using auxiliary mixture sampling (FrühwirthSchnatter and Wagner, 2006; Frühwirth-Schnatter and Frühwirth, 2007; Frühwirth-Schnatter et al., 2009). This allows variable selection for state space modelling of times series of small counts based on the Poisson distribution and of binary as well as categorical time 
series based on the logit transform. We provide an illustrative application to two time series of small counts.

\subsection{A Basic structural model for Count Data including Inter- vention}

For count data the basic structural model reads (Harvey and Durbin, 1986):

$$
\begin{aligned}
& y_{t} \sim \mathcal{P}\left(e_{t} \lambda_{t}\right), \\
& \log \lambda_{t}=\mu_{t}+s_{t}, \\
& \mu_{t}=\mu_{t-1}+a_{t-1}+\omega_{1 t}, \quad \omega_{1 t} \sim \mathcal{N}\left(0, \theta_{1}\right) \\
& a_{t}=a_{t-1}+\omega_{2 t}, \quad \omega_{2 t} \sim \mathcal{N}\left(0, \theta_{2}\right), \\
& s_{t}=-s_{t-1}-\cdots-s_{t-S+1}+\omega_{3 t}, \quad \omega_{3 t} \sim \mathcal{N}\left(0, \theta_{3}\right) .
\end{aligned}
$$

To account for the intervention at $t=t_{\text {int }}$, equation (38) is modified in the following way:

$$
\mu_{t}=\mu_{t-1}+a_{t-1}+\Delta+\omega_{1 t}
$$

\subsubsection{Stochastic model specification search}

Indicators $\delta, \delta_{3}, \gamma_{1}, \gamma_{2}$ and $\gamma_{3}$ are introduced as in Section 3 to select the structural components, and an additional indicator $\delta_{4}$ is introduced for the intervention effect. In the centered parameterization, equations (36) and (37) are modified in the following way:

$$
\begin{aligned}
& \log \lambda_{t}=\mu_{t}+\delta_{3} s_{0, q(t)}+\gamma_{3}\left(s_{t}-\delta_{3} s_{0, q(t)}\right), \\
& \mu_{t}=\mu_{t-1}+\delta a_{0}+\gamma_{2}\left(a_{t-1}-\delta a_{0}\right)+\delta_{4} I_{\left\{t=t_{i n t}\right\}} \Delta+\gamma_{1} \omega_{1 t},
\end{aligned}
$$

while (38) and (39) are unaffected. For MCMC estimation, the noncentered version of this model is required which reads:

$$
\log \lambda_{t}=\mu_{0}+\delta t a_{0}+\delta_{3} s_{0, q(t)}+\delta_{4} I_{\left\{t \geq t_{i n t}\right\}} \Delta+\gamma_{1} \sqrt{\theta_{1}} \tilde{\mu}_{t}+\gamma_{2} \sqrt{\theta_{2}} \tilde{A}_{t}+\gamma_{3} \sqrt{\theta_{3}} \tilde{s}_{t},
$$

where $\tilde{\mu}_{t}$ and $\tilde{A}_{t}$ are defined as in (11) to (13), while $\tilde{s}_{t}$ is defined as in (31). 


\subsubsection{MCMC Estimation}

MCMC estimation is implemented using auxiliary mixture sampling for count data. For each $t$, the distribution of $y_{t} \mid \lambda_{t}$ is regarded as the distribution of the number of jumps of an unobserved Poisson process with intensity $e_{t} \lambda_{t}$, having occurred in the time interval $[0,1]$. Frühwirth-Schnatter and Wagner (2006) create such a Poisson process for each observation $y_{t}$ and introduce the $\left(y_{t}+1\right)$ interarrival times of this Poisson process as latent variables, yielding a total of $T+\sum_{t=1}^{T} y_{t}$ latent variables.

Frühwirth-Schnatter et al. (2009) suggest a more efficient method based on introducing for each observation $y_{t}$ at most two latent variables, namely the interarrival time $\tau_{t 1}$ between the $y_{t}$ th jump and the next one and, if $y_{t}>0$, the arrival time $\tau_{t 2}$ of the $y_{t}$ th jump. Since $\tau_{t 1} \sim \mathcal{E}\left(e_{t} \lambda_{t}\right)$ and $\tau_{t 2} \sim \mathcal{G}\left(y_{t}, e_{t} \lambda_{t}\right)$ we have for $j=1,1+\min \left(y_{t}, 1\right)$ :

$$
-\log \tau_{t j}=\log e_{t}+\log \lambda_{t}+\varepsilon_{t j}
$$

where $\varepsilon_{t j}=-\log \xi_{t j}$ and $\xi_{t j} \sim \mathcal{G}\left(\nu_{t j}, 1\right)$ with integer shape parameter equal to $\nu_{t j}=$ $\max \left(1,(j-1) y_{t}\right)$. The distribution of the negative log Gamma distribution is approximated in Frühwirth-Schnatter et al. (2009) for each integer value $\nu$ by a mixture of normal distributions with component indicator $r_{t j}$ :

$$
p_{\varepsilon}\left(\varepsilon_{t j} ; \nu_{t j}\right)=\frac{\exp \left(-\nu_{t j} \varepsilon_{t j}-e^{-\varepsilon_{t j}}\right)}{\Gamma\left(\nu_{t j}\right)} \approx \sum_{r_{t j}=1}^{R\left(\nu_{t j}\right)} w_{r_{t j}}\left(\nu_{t j}\right) f_{N}\left(\varepsilon_{t j} ; m_{r_{t j}}\left(\nu_{t j}\right), s_{r_{t j}}^{2}\left(\nu_{t j}\right)\right) .
$$

The number of components $R(\nu)$ depends on $\nu$, as do the weights $w_{r}(\nu)$, the means $m_{r}(\nu)$ and the variances $s_{r}^{2}(\nu)$, see Frühwirth-Schnatter et al. (2009, Appendix A) for more details.

Introducing the auxiliary variables $\mathbf{u}=\left(\mathbf{u}_{1}, \ldots, \mathbf{u}_{T}\right)$, where $\mathbf{u}_{t}=\left(\tau_{t j}, r_{t j}, j=1,1+\right.$ $\left.\min \left(y_{t}, 1\right)\right)$, leads to a conditionally Gaussian state space model:

$$
\begin{aligned}
-\log \tau_{t j} & =\log e_{t}+\mu_{0}+\delta t a_{0}+\delta_{3} s_{0, q(t)}+\delta_{4} I_{\left\{t \geq t_{i n t}\right\}} \Delta \\
& +\gamma_{1} \sqrt{\theta_{1}} \tilde{\mu}_{t}+\gamma_{2} \sqrt{\theta_{2}} \tilde{A}_{t}+\gamma_{3} \sqrt{\theta_{3}} \tilde{s}_{t}+m_{r_{t j}}\left(\nu_{t j}\right)+\varepsilon_{t j}, \quad \varepsilon_{t j} \sim \mathcal{N}\left(0, s_{r_{t j}}^{2}\left(\nu_{t j}\right)\right) .
\end{aligned}
$$

In (43) we are dealing with a state space model that is conditionally Gaussian with the state vector $\mathbf{x}_{t}$ being the same as in Subsection 3.2. The MCMC scheme introduced in Subsection 3.2 for Gaussian state space models needs only a few modifications. First, an 
additional step has to be added to draw the auxiliary variables $\mathbf{u}$. Second, conditional on the state vector, we are dealing with a regression model with heteroscedastic normal errors with known error variance:

$$
\tilde{\mathbf{y}}=\mathbf{Z}^{\delta, \gamma} \boldsymbol{\beta}^{\delta, \gamma}+\varepsilon, \quad \varepsilon \sim \mathcal{N}(\mathbf{0}, \boldsymbol{\Sigma})
$$

where $\tilde{\mathbf{y}}$ denotes the collection of the auxiliary variables $\left(-\log \tau_{t j}-m_{r_{t j}}\left(\nu_{t j}\right)-\log e_{t}\right)$ and $\boldsymbol{\Sigma}$ is a diagonal matrix with elements $s_{r_{t j}}^{2}\left(\nu_{t j}\right)$. Under the normal prior $\boldsymbol{\beta}^{\boldsymbol{\delta}, \boldsymbol{\gamma}} \sim$ $\mathcal{N}\left(\mathbf{a}_{0}^{\boldsymbol{\delta}, \boldsymbol{\gamma}}, \mathbf{A}_{0}^{\boldsymbol{\delta}, \boldsymbol{\gamma}}\right)$, the marginal likelihood in this regression model defines $p(\mathbf{y} \mid \boldsymbol{\delta}, \boldsymbol{\gamma}, \mathbf{x}, \mathbf{u})$ :

$$
\begin{aligned}
& p(\mathbf{y} \mid \boldsymbol{\delta}, \boldsymbol{\gamma}, \mathbf{x}, \mathbf{u}) \\
& =\frac{|\boldsymbol{\Sigma}|^{-1 / 2}\left|\mathbf{A}_{T}^{\boldsymbol{\delta}, \boldsymbol{\gamma}}\right|^{1 / 2}}{(2 \pi)^{T / 2}\left|\mathbf{A}_{0}^{\boldsymbol{\delta}, \boldsymbol{\gamma}}\right|^{1 / 2}} \exp \left(-\frac{1}{2}\left(\tilde{\mathbf{y}}^{\prime} \boldsymbol{\Sigma}^{-1} \tilde{\mathbf{y}}+\left(\mathbf{a}_{0}^{\boldsymbol{\delta}, \boldsymbol{\gamma}}\right)^{\prime}\left(\mathbf{A}_{0}^{\boldsymbol{\delta}, \boldsymbol{\gamma}}\right)^{-1} \mathbf{a}_{0}^{\boldsymbol{\delta}, \boldsymbol{\gamma}}-\left(\mathbf{a}_{T}^{\boldsymbol{\delta}, \boldsymbol{\gamma}}\right)^{\prime}\left(\mathbf{A}_{T}^{\boldsymbol{\delta}, \boldsymbol{\gamma}}\right)^{-1} \mathbf{a}_{T}^{\boldsymbol{\delta}, \boldsymbol{\gamma}}\right)\right),
\end{aligned}
$$

where

$$
\begin{aligned}
& \left(\mathbf{A}_{T}^{\boldsymbol{\delta}, \boldsymbol{\gamma}}\right)^{-1}=\left(\left(\mathbf{Z}^{\boldsymbol{\delta}, \boldsymbol{\gamma}}\right)^{\prime} \boldsymbol{\Sigma}^{-1} \mathbf{Z}^{\boldsymbol{\delta}, \boldsymbol{\gamma}}+\left(\mathbf{A}_{0}^{\boldsymbol{\delta}, \boldsymbol{\gamma}}\right)^{-1}\right), \\
& \mathbf{a}_{T}^{\boldsymbol{\delta}, \boldsymbol{\gamma}}=\mathbf{A}_{T}^{\boldsymbol{\delta}, \boldsymbol{\gamma}}\left(\left(\mathbf{Z}^{\boldsymbol{\delta}, \boldsymbol{\gamma}}\right)^{\prime} \boldsymbol{\Sigma}^{-1} \tilde{\mathbf{y}}+\left(\mathbf{A}_{0}^{\boldsymbol{\delta}, \boldsymbol{\gamma}}\right)^{-1} \mathbf{a}_{0}^{\boldsymbol{\delta}, \boldsymbol{\gamma}}\right) .
\end{aligned}
$$

The MCMC scheme reads:

(a1) Sample $\boldsymbol{\delta}$ and $\boldsymbol{\gamma}$ from $p(\boldsymbol{\delta}, \boldsymbol{\gamma} \mid \mathbf{x}, \mathbf{u}, \mathbf{y}) \propto p(\mathbf{y} \mid \boldsymbol{\delta}, \boldsymbol{\gamma}, \mathbf{x}, \mathbf{u}) p(\boldsymbol{\delta}, \boldsymbol{\gamma})$ conditional on the state process $\mathbf{x}$ and the auxiliary variables $\mathbf{u}$ using the marginal likelihood (45) obtained from regression model (43).

(a2) Sample all unrestricted elements of the initial values of $\mathbf{x}_{0}$ and all unrestricted variance parameters $\sqrt{\theta_{i}}$ jointly from the multivariate normal distribution $\mathcal{N}\left(\mathbf{a}_{T}^{\boldsymbol{\delta}, \boldsymbol{\gamma}}, \mathbf{A}_{T}^{\boldsymbol{\delta}, \boldsymbol{\gamma}}\right)$ conditional on $\mathbf{x}$ and $\mathbf{u}$ using the moments (46) and (47); set all remaining initial values of $\mathbf{x}_{0}$ and all remaining variances equal to 0 .

(b) Sample $\mathbf{x}=\left(\mathbf{x}_{1}, \ldots, \mathbf{x}_{T}\right)$ from an appropriate state space form;

(c) Perform random sign switches as in step (c) in Subsection 3.2.

(d) Sample the auxiliary variables $\mathbf{u}$ conditional on the current risk $\lambda_{1}, \ldots, \lambda_{T}$ as in Frühwirth-Schnatter et al. (2009) for each $t=1, \ldots, T$ :

(d1) Sample $\xi_{t} \sim \mathcal{E}\left(e_{t} \lambda_{t}\right)$. If $y_{t}=0$, set $\tau_{t 1}=1+\xi_{t}$. If $y_{t}>0$, sample $\tau_{t 2}$ from a $\mathcal{B}\left(y_{t}, 1\right)$-distribution and set $\tau_{t 1}=1-\tau_{t 2}+\xi_{t}$. 
(d2) Sample the component indicator $r_{t j}$ for $j=1,1+\min \left(y_{t}, 1\right)$ from the following discrete distribution where $k=1, \ldots, R\left(\nu_{t j}\right)$

$$
\operatorname{Pr}\left(r_{t j}=k \mid \tau_{t j}, \lambda_{t}\right) \propto w_{k}\left(\nu_{t j}\right) f_{N}\left(-\log \tau_{i j}-\log \lambda_{t}-\log e_{t} ; m_{k}\left(\nu_{t j}\right), s_{k}^{2}\left(\nu_{t j}\right)\right) .
$$

Note that in step (a) marginalizing over the variables and components which are subject to model selection would not be possible for non-Gaussian state space models without the use of auxiliary mixture sampling or another augmentation scheme that leads to a conditionally Gaussian model. Such data augmentation schemes which enable variable selection in non-Gaussian models have been applied earlier by Holmes and Held (2006) for binary and multinomial regression model and by Tüchler (2008) for binary and multinomial regression models with random effects.

The partially proper normal prior and the fractional prior considered in Subsection 3.3 are easily adjusted for non-Gaussian state space models. A partially proper normal prior combines $p\left(\mu_{0}\right) \propto 1$ with a proper prior $\mathcal{N}\left(\mathbf{0}, \mathbf{B}_{0}^{\boldsymbol{\delta}, \boldsymbol{\gamma}}\right)$ on the remaining unrestricted elements of $\boldsymbol{\beta}^{\boldsymbol{\delta}, \boldsymbol{\gamma}}$ where $\mathbf{B}_{0}^{\boldsymbol{\delta}, \boldsymbol{\gamma}}=B_{0} \mathbf{I}$ corresponds to $\mathbf{a}_{0}^{\boldsymbol{\delta}, \boldsymbol{\gamma}}=\mathbf{0}$ and $\mathbf{A}_{0}^{\boldsymbol{\delta}, \boldsymbol{\gamma}}$ being the same as in (34). The marginal likelihood for this prior reads

$$
p(\mathbf{y} \mid \boldsymbol{\delta}, \boldsymbol{\gamma}, \mathbf{x}, \mathbf{u})=\frac{|\boldsymbol{\Sigma}|^{-1 / 2}\left|\mathbf{A}_{T}^{\boldsymbol{\delta}, \boldsymbol{\gamma}}\right|^{1 / 2}}{(2 \pi)^{(T-1) / 2}\left|\mathbf{B}_{0}^{\boldsymbol{\delta}, \boldsymbol{\gamma}}\right|^{1 / 2}} \cdot \exp \left(-\frac{1}{2}\left(\tilde{\mathbf{y}}^{\prime} \boldsymbol{\Sigma}^{-1} \tilde{\mathbf{y}}-\left(\mathbf{a}_{T}^{\boldsymbol{\delta}, \boldsymbol{\gamma}}\right)^{\prime}\left(\mathbf{A}_{T}^{\boldsymbol{\delta}, \boldsymbol{\gamma}}\right)^{-1} \mathbf{a}_{T}^{\boldsymbol{\delta}, \boldsymbol{\gamma}}\right)\right) .
$$

For a fractional prior, derived as in Subsection 3.3, the marginal likelihood is given as

$$
p(\mathbf{y} \mid \boldsymbol{\delta}, \boldsymbol{\gamma}, \mathbf{x}, \mathbf{u})=b^{q / 2}\left(\frac{|\boldsymbol{\Sigma}|^{-1}}{(2 \pi)^{T}}\right)^{(1-b) / 2} \cdot \exp \left(-\frac{(1-b)}{2}\left(\tilde{\mathbf{y}}^{\prime} \boldsymbol{\Sigma}^{-1} \tilde{\mathbf{y}}-\left(\mathbf{a}_{T}^{\boldsymbol{\delta}, \boldsymbol{\gamma}}\right)^{\prime}\left(\mathbf{A}_{T}^{\boldsymbol{\delta}, \boldsymbol{\gamma}}\right)^{-1} \mathbf{a}_{T}^{\boldsymbol{\delta}, \boldsymbol{\gamma}}\right)\right),
$$

where $\left(\mathbf{A}_{T}^{\boldsymbol{\delta}, \boldsymbol{\gamma}}\right)^{-1}=\left(\mathbf{Z}^{\boldsymbol{\delta}, \boldsymbol{\gamma}}\right)^{\prime} \boldsymbol{\Sigma}^{-1} \mathbf{Z}^{\boldsymbol{\delta}, \boldsymbol{\gamma}}$ and $\mathbf{a}_{T}^{\boldsymbol{\delta}, \boldsymbol{\gamma}}=\mathbf{A}_{T}^{\boldsymbol{\delta}, \boldsymbol{\gamma}}\left(\mathbf{Z}^{\boldsymbol{\delta}, \boldsymbol{\gamma}}\right)^{\prime} \boldsymbol{\Sigma}^{-1} \tilde{\mathbf{y}}$

\subsection{Road Safety Data}

We analyze a time series consisting of monthly counts of killed or injured pedestrians, aged 6-10, from 1987-2005 in Linz, which is the third largest town in Austria. ${ }^{2}$ The observations are a series of small counts not exceeding 5 , see Figure 6 . A new law intended to increase

\footnotetext{
${ }^{2}$ A shorter version of this time series ranging from 1987-2002 was analyzed in Frühwirth-Schnatter and Wagner (2006).
} 
road safety came into force in Austria on October 1, 1994, since when pedestrians who want to use a pedestrian crossing have to be allowed to cross. Of interest is the effect of this law on the (monthly) risk of being killed or seriously injured in a road accident as a child living in Linz.

The basic structural model with intervention effect for Poisson counts defined in Subsection 4.1 is fitted to the number $y_{t}$ of children killed or seriously injured in time period $t, y_{t} \sim \mathcal{P}\left(e_{t} \lambda_{t}\right)$, where $e_{t}$ is the number of children living in Linz. Model specification search is carried out to identify an appropriate model.

\subsubsection{Bayesian Inference for the unrestricted basic structural model with intervention}

For illustration, we start with Bayesian inference without variable selection for the unrestricted basic structural model with intervention. For these data, we were not able to estimate the model under the completely centered parameterization as MCMC did not converge. For this reason, we compare a parameterization where only the season is noncentered (Frühwirth-Schnatter and Wagner, 2006) under the priors $\theta_{i} \sim \mathcal{G}^{-1}(0.1,0.001), i=$ $1,2, \pm \sqrt{\theta_{3}} \sim \mathcal{N}(0,1)$ with a fully noncentered model with priors $\pm \sqrt{\theta_{i}} \sim \mathcal{N}(0,1), i=$ $1,2,3$. For both parameterizations we assume that $\mu_{0} \sim \mathcal{N}\left(\log \left(y_{1} / e_{1}\right), 1\right)=\mathcal{N}(-9.0084,1)$ and that the unknown initial values of the other components and the intervention effect follow a standard normal prior distribution.

Figure 8 shows histograms of the MCMC draws for $\pm \sqrt{\theta_{i}}, i=1, \ldots, 3$ for both priors. For the normal prior the posterior of all parameters $\pm \sqrt{\theta_{i}}, i=1, \ldots, 3$ is clearly centered at 0 , suggesting that the state space model is overfitting and the data may be explained by a simply Poisson regression model. This finding is confirmed by stochastic model specification search in Subsection 4.2.2. As in Subsection 3.4.1 the inverted Gamma density is very influential and shrinks the posterior densities of $\pm \sqrt{\theta_{1}}$ and $\pm \sqrt{\theta_{2}}$ away from 0 , spuriously suggesting that $\theta_{1}>0$ and $\theta_{2}>0$.

Figure 9 shows the various components of the unconstrained model like the smoothed level $\mu_{t}$ with pointwise $95 \%$ credibility intervals. The estimated monthly risk $\lambda_{t}$ for a child to be seriously injured or killed seems to decrease at the time of intervention. The drift $a_{t}$ is not significantly different from 0 over the whole observation period. The seasonal 
component has significantly lower values than the annual average in the holiday months July and August and higher values in June and October.

We used 20,000 iterations after a burn-in of 5,000 for each parameterization. As in Subsection 3.4.1, we observe much better mixing behavior of the MCMC sampler under the non-centered parametrization, see Figure 7.

\subsubsection{Stochastic Model Specification Search}

Stochastic model specification search is carried out using the prior described in Subsection 3.3. We compare partially proper priors with different prior variances $B_{0}$ with fractional priors with different fractions $b$. For each prior, MCMC sampling was carried out for $M=100,000$ draws after a burn-in of 20,000 draws. The first 1000 draws of the burn-in were drawn from the unrestricted model, model selection began after these first 1000 draws. Depending on the prior, running MCMC takes between 125 and 165 minutes, see also Table 5 .

Results of the variable selection procedure are summarized in Table 4 and 5 . The most frequently visited model is fairly robust against the choice of the prior, only the partially proper prior with the largest $B_{0}$ and the fractional prior with the smallest $b$ lead to a more parsimonious model. No trend is present in the selected model, because $\delta=0$ and $\gamma_{2}=0$ imply that $a_{t}=a_{0}=0$ for the whole observation period. The initial seasonal pattern is significant $\left(\delta_{3}=1\right)$, but does not change over time $\left(\gamma_{3}=0\right)$. The level of the model is constant before and after intervention, because $\gamma_{1}=0$. Most importantly, the intervention effect is significant, because $\delta_{4}=1$ is selected. Interestingly, the selected model is no longer a state space model $\left(\gamma_{1}=\gamma_{2}=\gamma_{3}=0\right)$, but a simple Poisson regression model with monthly seasonal dummies and an intervention effect. This finding is confirmed by the marginal likelihoods computed in Frühwirth-Schnatter and Wagner (2008).

In Table 6 and Figure 10, we compare posterior inference for the intervention effect for the unconstrained basic structural model and the model obtained by variable selection. We observe here an impressive gain of statistical efficiency for this parameter of interest. For the unconstrained basic structural model, making the level dynamic before and after 
the intervention causes quite a loss of information, leading to an intervention effect that is not significant.

The seasonal pattern disappears, if $B_{0}$ is rather large in the partially proper prior and if $b$ is rather small in the fractional prior, see Table 4 and 5 . Increasing $B_{0}$ and decreasing $b$ forces more parsimonious models. Not surprisingly the seasonal pattern disappears first in a more parsimonious model, because in the selected model only a few seasonal dummies are different from 0, see also Figure 9.

\subsection{Purse snatching in Hyde Park, Chicago}

For further illustration, we reanalyze a time series of cases of purse snatching $y_{t}$ in the Hyde park neighborhood in Chicago (Harvey, 1989) reported for the period from January 1968 to September 1973. We consider a simplified version of the model introduced in Subsection 4.1, where no seasonal and no intervention effect is present and the exposures $e_{t}$ are equal to 1.

First, Gibbs sampling was run without variable selection for 15,000 iterations after a burn-in of 10,000 . We selected the normal prior $\mathcal{N}(0,10)$ both for $\mu_{0}$ and $a_{0}$ and compare the inverted Gamma prior $\theta_{i} \sim \mathcal{G}^{-1}(-0.5,0.0001)$ with the normal prior $\pm \sqrt{\theta_{i}} \sim \mathcal{N}(0,1)$, for $i=1,2$. Figure 11 shows histograms of $\pm \sqrt{\theta_{i}}$ under both priors. The posterior for $\pm \sqrt{\theta_{1}}$ is roughly the same under both priors and clearly indicates that $\theta_{1}>0$. Again, the inverted Gamma density is too influential for $\theta_{2}$ and shrinks the draws away from 0 , while for the normal prior the posterior is clearly centered at 0 , suggesting that $\theta_{2}=0$.

Second, stochastic model specification search is carried out using the prior described in Subsection 3.3. We compare partially proper priors with different prior variances $B_{0}$ with fractional priors with different fractions $b$. For each prior, MCMC sampling was carried out for $M=100,000$ draws after a burn-in of 20,000 draws. The first 1000 draws of the burn-in were drawn from the unrestricted model, model selection began after these first 1000 draws. Depending on the prior, running MCMC takes between 25 and 28 minutes, see also Table 8.

Results of the variable selection procedure are presented in Table 7 and 8. Model selection is extremely robust to the prior choice and clearly picks a local level model. The 
drift disappears because $\delta=0$ and $\gamma_{2}=0$ imply that $a_{t} \equiv a_{0}=0$ for all $t$. This finding confirms model selection by the marginal likelihoods in Frühwirth-Schnatter and Wagner (2008).

\section{Concluding remarks}

The model space MCMC approach discussed in this paper could be easily adapted to other state space models. An important extension we are investigating currently is searching for fixed and time-varying coefficients in a regression model.

It is possible to extend our approach to several other non-Gaussian time series models. The method works for any partial Gaussian state space model in the sense of Shephard (1994), i.e. for any state space model that is conditionally Gaussian given a set of auxiliary latent variables. Thus it is straightforward to extend the approach to robust state space modeling based on the Student- $t$ distribution as discussed e.g. in Carlin, Polson, and Stoffer (1992) or to modelling binary time series based on probit-link state space models as considered e.g. in Czado and Song (2008).

Auxiliary mixture sampling as discussed in Frühwirth-Schnatter and Frühwirth (2007) allows to consider state space modelling of binary and categorical time series based on the logit link. Frühwirth-Schnatter et al. (2009) shows how to run auxiliary mixture sampling for data from the negative binomial distribution. In combination with the idea of the present paper, this allows variable selection for time series of overdispered count data. Furthermore, Frühwirth-Schnatter et al. (2009) discuss efficient auxiliary mixture sampling for data from the binomial and the multinomial distribution where the dimension of the auxiliary variable $\mathbf{u}_{t}$ is limited to $2 m$, with $m+1$ being the number of alternatives, even if the number $y_{t}$ of observed counts is increasing. This makes it feasible to extend variable selection to state space modelling of binomial and multinomial data.

A couple of modifications of our approach are worth being considered. First, the uniform prior over all models may be substituted by a more flexible prior which is obtained by assuming that the prior occurrence of $\delta_{i}=1$ and $\gamma_{i}=1$ is different:

$$
\operatorname{Pr}\left(\delta_{i}=1 \mid \alpha_{\delta}\right)=\alpha_{\delta}, \quad \operatorname{Pr}\left(\gamma_{i}=1 \mid \alpha_{\gamma}\right)=\alpha_{\gamma} .
$$


In this prior, $\alpha_{\delta}$ and $\alpha_{\gamma}$ may be chosen as fixed values, if prior information on the occurrence probabilities is available. If this is not the case, a hyperprior may be put on $\alpha_{\delta}$ and $\alpha_{\gamma}$ as in Smith and Kohn (2002) and Frühwirth-Schnatter and Tüchler (2008). If both hyperparameters $\alpha_{\delta}$ and $\alpha_{\gamma}$ are iid Uniform on $[0,1]$, then

$$
p(\boldsymbol{\delta}, \boldsymbol{\gamma})=B\left(1+\sum_{i} I_{\left\{\delta_{i}=1\right\}}, 1+\sum_{i} I_{\left\{\delta_{i}=0\right\}}\right) B\left(1+\sum_{i} I_{\left\{\gamma_{i}=1\right\}}, 1+\sum_{i} I_{\left\{\gamma_{i}=0\right\}}\right),
$$

where $B(\cdot, \cdot)$ is the Beta function. This prior leads to a uniform distribution over model size and outperforms the uniform prior over all models in variable selection for large regression models, see Ley and Steel (2007). In our applications, where model size is small, posterior inference under both priors is virtually the same.

Second, sampling the indicators could be modified. In our MCMC schemes, the indicators $(\boldsymbol{\delta}, \boldsymbol{\gamma})$ are sampled jointly from the discrete posterior $p(\boldsymbol{\delta}, \boldsymbol{\gamma} \mid \mathbf{x}, \mathbf{y})$ by evaluating the right hand side of (25) for all combinations of indicators at each sweep of the sampler. This multi-move sampling is rather time-consuming and may be substituted by single-move Gibbs sampling, i.e. sampling recursively from $p\left(\delta_{j} \mid \boldsymbol{\delta}_{-j}, \boldsymbol{\gamma}, \mathbf{x}, \mathbf{y}\right)$ and $p\left(\gamma_{j} \mid \boldsymbol{\gamma}_{-j}, \boldsymbol{\delta}, \mathbf{x}, \mathbf{y}\right)$ as in George and McCulloch (1993).

An open issue of our approach is the influence the prior on the initial values and the process variances exercises on final model selection. We demonstrated that the normal prior put on the signed square root of the process variances is far less influential than the usual inverted Gamma for the process variances themselves. The sensitivity analysis carried out for all of our case studies revealed a surprising robustness of the finally selected model against variation in the normal prior. A concise statement which prior scale leads to model consistency in the sense of Casella, Girón, Martínez, and Moreno (2009), however, is far beyond the scope of the present paper.

\section{References}

Carlin, B. P., N. G. Polson, and D. S. Stoffer (1992). A Monte Carlo approach to nonnormal and nonlinear state-space modeling. Journal of the American Statistical Association 87, 493-500.

Carter, C. K. and R. Kohn (1994). On Gibbs sampling for state space models. Biometrika 81, 541-553. 
Casella, G., F. J. Girón, M. L. Martínez, and E. Moreno (2009). Consistency of Bayesian procedures for variable selection. The Annals of Statistcs 37, 1207-1228.

Chen, Z. and D. Dunson (2003). Random effects selection in linear mixed models. Biometrics 59, 762-769.

Chib, S. (1995). Marginal likelihood from the Gibbs output. Journal of the American Statistical Association 90, 1313-1321.

Czado, C. and P. X.-K. Song (2008). State space mixed models for longitudinal observations with binary and binomial responses. Statistical Papers 49, 691-714.

De Jong, P. and N. Shephard (1995). The simulation smoother for time series models. Biometrika 82, 339-350.

Durbin, J. and S. J. Koopman (2000). Time series analysis of non-Gaussian observations based on state space models from both classical and Bayesian perspectives. Journal of the Royal Statistical Society, Ser. B 62, 3-56.

Durbin, J. and S. J. Koopman (2001). Time Series Analysis by State Space Methods. Oxford: Oxford University Press.

Fernández, C., E. Ley, and M. F. J. Steel (2001). Benchmark priors for Bayesian model averaging. Journal of Econometrics 100, 381-427.

Frühwirth-Schnatter, S. (1994). Data augmentation and dynamic linear models. Journal of Time Series Analysis 15, 183-202.

Frühwirth-Schnatter, S. (1995). Bayesian model discrimination and Bayes factors for linear Gaussian state space models. Journal of the Royal Statistical Society, Ser. B 57, $237-246$.

Frühwirth-Schnatter, S. (2001). Fully Bayesian analysis of switching Gaussian state space models. Annals of the Institute of Statistical Mathematics 53, 31-49.

Frühwirth-Schnatter, S. (2004). Efficient Bayesian parameter estimation for state space models based on reparameterizations. In A. Harvey, S. J. Koopman, and N. Shephard 
(Eds.), State Space and Unobserved Component Models: Theory and Applications. Proceedings of a Conference in Honour of James Durbin, pp. 123-151. Cambridge: Cambridge University Press.

Frühwirth-Schnatter, S. and R. Frühwirth (2007). Auxiliary mixture sampling with applications to logistic models. Computational Statistics and Data Analysis 51, 3509-3528.

Frühwirth-Schnatter, S., R. Frühwirth, L. Held, and H. Rue (2009). Improved auxiliary mixture sampling for hierarchical models of non-Gaussian data. Statistics and Computing 19, forthcoming.

Frühwirth-Schnatter, S. and R. Tüchler (2008). Bayesian parsimonious covariance estimation for hierarchical linear mixed models. Statistics and Computing 18, 1-13.

Frühwirth-Schnatter, S. and H. Wagner (2006). Auxiliary mixture sampling for parameterdriven models of time series of counts with applications to state space modelling. Biometrika 93, 827-841.

Frühwirth-Schnatter, S. and H. Wagner (2008). Marginal likelihoods for non-Gaussian models using auxiliary mixture sampling. Computational Statistics and Data Analysis 52, 4608-4624.

Gelman, A. (2006). Prior distributions for variance parameters in hierarchical models. Bayesian Analysis 1, 515-534.

George, E. I. and R. McCulloch (1993). Variable selection via Gibbs sampling. Journal of the American Statistical Association 88, 881-889.

George, E. I. and R. McCulloch (1997). Approaches for Bayesian variable selection. Statistica Sinica 7, 339-373.

Geweke, J. (1996). Variable selection and model comparison in regression. In J. M. Bernardo, J. O. Berger, A. P. Dawid, and A. F. M. Smith (Eds.), Bayesian Statistics 5 Proceedings of the Fifth Valencia International Meeting, pp. 609-620. Oxford University Press.

Green, P. J. (1995). Reversible jump Markov chain Monte Carlo computation and Bayesian model determination. Biometrika 82, 711-732. 
Harvey, A. C. (1989). Forecasting, Structural Time Series Models and the Kalman Filter. Cambridge: Cambridge University Press.

Harvey, A. C. and J. Durbin (1986). The effects of seat belt legislation on British road casualties: A case study in structural time series modelling. Journal of the Royal Statistical Society, Ser. A 149, 187-227.

Holmes, C. C. and L. Held (2006). Bayesian auxiliary variable models for binary and multinomial regression. Bayesian Analysis 1, 145-168.

Jeffreys, S. H. (1948). Theory of Probability (2 ed.). Oxford: Clarendon.

Ley, E. and M. F. J. Steel (2009). On the effect of prior assumptions in Bayesian model averaging with applications to growth regression. Journal of Applied Econometrics 24, $651-674$

O'Hagan, A. (1995). Fractional Bayes factors for model comparison (Disc: p118-138). Journal of the Royal Statistical Society, Ser. B 57, 99-118.

Pitt, M. K. and N. Shephard (1999). Analytic convergence rates and parameterization issues for the Gibbs sampler applied to state space models. Journal of Time Series Analysis 20, 63-85.

Shephard, N. (1994). Partial non-Gaussian state space. Biometrika 81, 115-131.

Shively, T. S. and R. Kohn (1997). A Bayesian approach to model selection in stochastic coefficient regression models and structural time series models. Journal of Econometrics $76,39-52$.

Shively, T. S., R. Kohn, and S. Wood (1999). Variable selection and function estimation in additive nonparametric regression using a data-based prior. Journal of the American Statistical Association 94, 777-794.

Smith, M. and R. Kohn (1996). Nonparametric regression using Bayesian variable selection. Journal of Econometrics 75, 317-343.

Smith, M. and R. Kohn (2002). Parsimonious covariance matrix estimation for longitudinal data. Journal of the American Statistical Association 97, 1141-1153. 
Tüchler, R. (2008). Bayesian variable selection for logistic models using auxiliary mixture sampling. Journal of Computational and Graphical Statistics 17, 76-94.

Zellner, A. (1971). An Introduction to Bayesian Inference in Econometrics. New York: Wiley. 
Table 1: UK coal consumption; the three most frequently visited models (among 100000 MCMC iterations) for various prior distributions

\begin{tabular}{|c|c|c|c|c|c|c|}
\hline prior & $\delta$ & $\delta_{3}$ & $\gamma_{1}$ & $\gamma_{2}$ & $\gamma_{3}$ & frequency \\
\hline \multirow[t]{3}{*}{$p\left(\mu_{0}\right) \propto 1, B_{0}=1$} & 0 & 1 & 0 & 1 & 0 & 44825 \\
\hline & 1 & 1 & 1 & 0 & 0 & 16323 \\
\hline & 0 & 1 & 0 & 1 & 1 & 11803 \\
\hline \multirow[t]{3}{*}{$p\left(\mu_{0}\right) \propto 1, B_{0}=10$} & 0 & 1 & 0 & 1 & 0 & 64018 \\
\hline & 1 & 1 & 1 & 0 & 0 & 14267 \\
\hline & 0 & 1 & 1 & 0 & 0 & 10655 \\
\hline \multirow[t]{3}{*}{$p\left(\mu_{0}\right) \propto 1, B_{0}=100$} & 0 & 1 & 0 & 1 & 0 & 74724 \\
\hline & 0 & 1 & 1 & 0 & 0 & 15597 \\
\hline & 1 & 1 & 1 & 0 & 0 & 5784 \\
\hline \multirow[t]{3}{*}{$b=10^{-3}$} & 0 & & 0 & 1 & 0 & 64205 \\
\hline & 0 & 1 & 1 & 1 & 0 & 10780 \\
\hline & 1 & 1 & 0 & 1 & 0 & 9033 \\
\hline \multirow[t]{3}{*}{$b=10^{-4}$} & 0 & 1 & 0 & 1 & 0 & 86097 \\
\hline & 1 & 1 & 0 & 1 & 0 & 4570 \\
\hline & 0 & 1 & 1 & 1 & 0 & 4291 \\
\hline \multirow[t]{3}{*}{$b=10^{-5}$} & 0 & 1 & 0 & 1 & 0 & 92323 \\
\hline & 0 & 1 & 1 & 0 & 0 & 3084 \\
\hline & 0 & 1 & 1 & 1 & 0 & 1717 \\
\hline
\end{tabular}

Table 2: UK coal consumption; posterior inclusion probability for each indicator under various priors

\begin{tabular}{|c|ccccc|c|}
\hline Prior & $\delta$ & $\delta_{3}$ & $\gamma_{1}$ & $\gamma_{2}$ & $\gamma_{3}$ & time $(\mathrm{min})$ \\
\hline$p\left(\mu_{0}\right) \propto 1, B_{0}=1$ & 0.2933 & $\mathbf{1 . 0 0 0 0}$ & 0.3712 & $\mathbf{0 . 7 5 0 0}$ & 0.2223 & 52.2 \\
$p\left(\mu_{0}\right) \propto 1, B_{0}=10$ & 0.1716 & $\mathbf{1 . 0 0 0 0}$ & 0.3136 & $\mathbf{0 . 7 4 1 0}$ & 0.0328 & 51.6 \\
$p\left(\mu_{0}\right) \propto 1, B_{0}=100$ & 0.0697 & $\mathbf{1 . 0 0 0 0}$ & 0.2344 & $\mathbf{0 . 7 8 3 6}$ & 0.0101 & 52.0 \\
\hline$b=10^{-3}$ & 0.1827 & $\mathbf{1 . 0 0 0 0}$ & 0.2154 & $\mathbf{0 . 9 2 1 0}$ & 0.0677 & 43.8 \\
$b=10^{-4}$ & 0.0630 & $\mathbf{1 . 0 0 0 0}$ & 0.0736 & $\mathbf{0 . 9 7 2 8}$ & 0.0216 & 43.6 \\
$b=10^{-5}$ & 0.0219 & $\mathbf{1 . 0 0 0 0}$ & 0.0600 & $\mathbf{0 . 9 5 7 6}$ & 0.0069 & 43.2 \\
\hline
\end{tabular}


Table 3: UK coal consumption; log marginal likelihoods $\log (\hat{p}(\mathbf{y} \mid \mathcal{M}))$ of all models $\mathcal{M}=\left(\delta, \delta_{3}, \gamma_{1}, \gamma_{2}, \gamma_{3}\right)$ estimated by importance sampling (standard errors in parenthesis); corresponding model probability $\hat{p}(\mathcal{M} \mid \mathbf{y})$ in comparison to the number $h(\mathcal{M})$ of times the model was visited during 100,000 MCMC iterations; $B_{0}=1$ (left hand side) and $B_{0}=100$ (right hand side)

\begin{tabular}{|c|c|c|c|c|c|c|}
\hline Model $\mathcal{M}$ & $\log (\hat{p}(\mathbf{y} \mid \mathcal{M}))$ & $\hat{p}(\mathcal{M} \mid \mathbf{y})$ & $h(\mathcal{M})$ & $\log (\hat{p}(\mathbf{y} \mid \mathcal{M})) \quad \hat{p}$ & $(\mathcal{M} \mid \mathbf{y})$ & $h(\mathcal{M})$ \\
\hline 0000000 & $-87.83(.001)$ & 0.000 & 0 & $-87.83(.001)$ & 0.000 & 0 \\
\hline $\begin{array}{lllll}0 & 0 & 0 & 0 & 1\end{array}$ & $-85.65(.001)$ & 0.000 & 0 & $-87.95(.001)$ & 0.000 & 0 \\
\hline 00010 & $-39.89(.002)$ & 0.000 & 0 & $-42.19(.002)$ & 0.000 & 0 \\
\hline $\begin{array}{llllll}0 & 0 & 0 & 1 & 1\end{array}$ & $16.32(.002)$ & 0.000 & 0 & $11.83(.002)$ & 0.000 & 0 \\
\hline 00100 & $-40.88(.001)$ & 0.000 & 0 & $-43.15(.001)$ & 0.000 & 0 \\
\hline $\begin{array}{lllll}0 & 0 & 1 & 0 & 1\end{array}$ & $14.60(.002)$ & 0.000 & 0 & $10.30(.002)$ & 0.000 & 0 \\
\hline 00110 & $-42.23(.007)$ & 0.000 & 0 & $-46.85(.005)$ & 0.000 & 0 \\
\hline $\begin{array}{lllll}0 & 0 & 1 & 1 & 1\end{array}$ & $15.00(.010)$ & 0.000 & 0 & $8.25(.006)$ & 0.000 & 0 \\
\hline 01000 & $-80.60(.001)$ & 0.000 & & $-87.00(.001)$ & 0.000 & 0 \\
\hline 01001 & $-83.73(.003)$ & 0.000 & 0 & $-92.42(.004)$ & 0.000 & 0 \\
\hline 0101010 & $28.68(.002)$ & 0.430 & 44013 & $26.67(.002)$ & 0.763 & 75099 \\
\hline 01011 & $27.42(.006)$ & 0.121 & 12074 & 22.06(.008) & 0.008 & 711 \\
\hline 01100 & $25.93(.001)$ & 0.027 & 2502 & 24.97(.002) & 0.139 & 14733 \\
\hline 011101 & $25.00(.005)$ & 0.011 & 926 & $20.56(.005)$ & 0.002 & 161 \\
\hline 011110 & 26.99(.006) & 0.079 & 8051 & 23.04(.006) & 0.020 & 2089 \\
\hline 01111 & $25.87(.010)$ & 0.026 & 2660 & $18.51(.010)$ & 0.000 & 33 \\
\hline 10000 & $-37.81(.001)$ & 0.000 & 0 & $-40.12(.001)$ & 0.000 & 0 \\
\hline $\begin{array}{lllll}1 & 0 & 0 & 0 & 1\end{array}$ & $8.05(.001)$ & 0.000 & 0 & $3.51(.001)$ & 0.000 & 0 \\
\hline 10010 & $-42.15(.004)$ & 0.000 & 0 & $-46.76(.003)$ & 0.000 & 0 \\
\hline 10011 & $14.33(.002)$ & 0.000 & 0 & $7.54(.002)$ & 0.000 & 0 \\
\hline 10100 & $-39.92(.004)$ & 0.000 & 0 & $-44.51(.005)$ & 0.000 & 0 \\
\hline 10101 & $16.26(.002)$ & 0.000 & 0 & $9.56(.002)$ & 0.000 & 0 \\
\hline 10110 & $-44.44(.005)$ & 0.000 & 0 & $-51.34(.005)$ & 0.000 & 0 \\
\hline $\begin{array}{lllll}1 & 0 & 1 & 1 & 1\end{array}$ & $13.39(.009)$ & 0.000 & 0 & $4.35(.009)$ & 0.000 & 0 \\
\hline 11000 & $20.86(.001)$ & 0.000 & 17 & $16.70(.001)$ & 0.000 & 1 \\
\hline 11001 & $18.63(.004)$ & 0.000 & 1 & $11.68(.003)$ & 0.000 & 0 \\
\hline 11010 & $26.50(.002)$ & 0.049 & 4866 & $22.28(.001)$ & 0.009 & 907 \\
\hline 11011 & $25.24(.005)$ & 0.014 & 1308 & $17.66(.004)$ & 0.000 & 8 \\
\hline 11100 & $27.75(.001)$ & 0.169 & 16332 & $24.08(.001)$ & 0.057 & 6160 \\
\hline 11101 & $26.69(.006)$ & 0.059 & 5656 & $19.60(.007)$ & 0.001 & 76 \\
\hline 11110 & $25.05(.007)$ & 0.011 & 1209 & $18.96(.006)$ & 0.000 & 22 \\
\hline 11111 & $23.98(.010)$ & 0.004 & 385 & $14.46(.009)$ & 0.000 & 0 \\
\hline
\end{tabular}


Table 4: Road safety data; the three most frequently visited models (among 100,000 MCMC iterations) for various prior distributions

\begin{tabular}{|c|cccccc|c|}
\hline prior & $\delta$ & $\delta_{3}$ & $\delta_{4}$ & $\gamma_{1}$ & $\gamma_{2}$ & $\gamma_{3}$ & frequency \\
\hline$p\left(\mu_{0}\right) \propto 1, B_{0}=1$ & $\mathbf{0}$ & $\mathbf{1}$ & $\mathbf{1}$ & $\mathbf{0}$ & $\mathbf{0}$ & $\mathbf{0}$ & 93463 \\
& 0 & 1 & 1 & 0 & 0 & 1 & 2581 \\
& 0 & 1 & 1 & 1 & 0 & 0 & 1508 \\
\hline$p\left(\mu_{0}\right) \propto 1, B_{0}=10$ & $\mathbf{0}$ & $\mathbf{1}$ & $\mathbf{1}$ & $\mathbf{0}$ & $\mathbf{0}$ & $\mathbf{0}$ & 93571 \\
& 0 & 1 & 0 & 0 & 0 & 0 & 4163 \\
& 0 & 1 & 1 & 0 & 0 & 1 & 773 \\
\hline$p\left(\mu_{0}\right) \propto 1, B_{0}=100$ & $\mathbf{0}$ & $\mathbf{0}$ & $\mathbf{1}$ & $\mathbf{0}$ & $\mathbf{0}$ & $\mathbf{0}$ & 75054 \\
& 0 & 0 & 0 & 0 & 0 & 0 & 15607 \\
& 0 & 0 & 1 & 0 & 0 & 1 & 6770 \\
\hline$b=10^{-2}$ & $\mathbf{0}$ & $\mathbf{1}$ & $\mathbf{1}$ & $\mathbf{0}$ & $\mathbf{0}$ & $\mathbf{0}$ & 23349 \\
& 0 & 1 & 1 & 0 & 0 & 1 & 10035 \\
& 0 & 1 & 1 & 1 & 0 & 0 & 8564 \\
\hline$b=10^{-3}$ & $\mathbf{0}$ & $\mathbf{1}$ & $\mathbf{1}$ & $\mathbf{0}$ & $\mathbf{0}$ & $\mathbf{0}$ & 48612 \\
& 1 & 1 & 0 & 0 & 0 & 0 & 9368 \\
& 0 & 1 & 1 & 0 & 0 & 1 & 7414 \\
\hline$b=10^{-4}$ & $\mathbf{0}$ & $\mathbf{1}$ & $\mathbf{1}$ & $\mathbf{0}$ & $\mathbf{0}$ & $\mathbf{0}$ & 62113 \\
& 1 & 1 & 0 & 0 & 0 & 0 & 11616 \\
& 0 & 1 & 0 & 0 & 1 & 0 & 6756 \\
\hline$b=10^{-5}$ & $\mathbf{0}$ & $\mathbf{0}$ & $\mathbf{1}$ & $\mathbf{0}$ & $\mathbf{0}$ & $\mathbf{0}$ & 38446 \\
& 0 & 0 & 1 & 0 & 0 & 1 & 19121 \\
& 1 & 0 & 0 & 0 & 0 & 0 & 10108 \\
\hline
\end{tabular}


Table 5: Road safety data; posterior inclusion probability for each indicator

\begin{tabular}{|c|ccc|ccc|c|}
\hline & trend & season & intervention & \multicolumn{3}{|c|}{ process variances } & \\
prior & $\delta$ & $\delta_{3}$ & $\delta_{4}$ & $\gamma_{1}$ & $\gamma_{2}$ & $\gamma_{3}$ & time (min.) \\
\hline$p\left(\mu_{0}\right) \propto 1, B_{0}=1$ & 0.0026 & $\mathbf{1 . 0 0 0 0}$ & $\mathbf{0 . 9 7 7 5}$ & 0.0222 & 0.0003 & 0.0269 & 164.9 \\
$p\left(\mu_{0}\right) \propto 1, B_{0}=10$ & 0.0022 & $\mathbf{1 . 0 0 0 0}$ & $\mathbf{0 . 9 4 9 1}$ & 0.0114 & 0.0002 & 0.0090 & 143.0 \\
$p\left(\mu_{0}\right) \propto 1, B_{0}=100$ & 0.0020 & 0.0127 & $\mathbf{0 . 8 3 1 6}$ & 0.0055 & 0.0001 & 0.0738 & 143.5 \\
\hline$b=10^{-2}$ & 0.3160 & $\mathbf{1 . 0 0 0 0}$ & $\mathbf{0 . 7 6 6 2}$ & 0.3008 & 0.2804 & 0.2973 & 125.7 \\
$b=10^{-3}$ & 0.1982 & $\mathbf{1 . 0 0 0 0}$ & $\mathbf{0 . 7 4 1 6}$ & 0.1443 & 0.1499 & 0.1305 & 125.6 \\
$b=10^{-4}$ & 0.1519 & $\mathbf{1 . 0 0 0 0}$ & $\mathbf{0 . 7 1 1 1}$ & 0.0813 & 0.1009 & 0.0433 & 126.2 \\
$b=10^{-5}$ & 0.1643 & 0 & $\mathbf{0 . 5 9 4 5}$ & 0.0703 & 0.0958 & 0.3192 & 125.6 \\
\hline
\end{tabular}

Table 6: Road safety data; posterior inference for the intervention effect $\Delta$

\begin{tabular}{|c|ccc|}
\hline$\Delta$ & Mean & Std.dev. & 95\%H.P.D. regions \\
\hline Basic structural model & -0.4035 & 0.5067 & {$[-1.4024 ; 0.5855]$} \\
\hline Poisson regression model with seasonal dummies & -0.3594 & 0.0967 & {$[-0.5450 ;-0.1662]$} \\
\hline
\end{tabular}

Table 7: Purse snatching; the three most frequently visited models (among 100,000 MCMC iterations) for various prior distributions

\begin{tabular}{|rcc|rrr|rrr|}
\hline$\delta$ & $\gamma_{1}$ & $\gamma_{2}$ & $B_{0}=1$ & $B_{0}=10$ & $B_{0}=100$ & $b=10^{-3}$ & $b=10^{-4}$ & $b=10^{-5}$ \\
\hline $\mathbf{0}$ & $\mathbf{1}$ & $\mathbf{0}$ & 95108 & 99226 & 99509 & 56254 & 83737 & 93211 \\
1 & 1 & 0 & 3710 & 667 & 403 & 19750 & 7630 & 3301 \\
0 & 1 & 1 & 1117 & 93 & 88 & 18679 & 8083 & 3403 \\
\hline
\end{tabular}


Table 8: Purse snatching; posterior inclusion probability for each indicator

\begin{tabular}{|c|ccc|c|}
\hline prior & $\delta$ & $\gamma_{1}$ & $\gamma_{2}$ & time (min.) \\
\hline$p\left(\mu_{0}\right) \propto 1, B_{0}=1$ & 0.0377 & $\mathbf{1 . 0 0 0 0}$ & 0.0118 & 27.8 \\
$p\left(\mu_{0}\right) \propto 1, B_{0}=10$ & 0.0068 & $\mathbf{1 . 0 0 0 0}$ & 0.0011 & 27.8 \\
$p\left(\mu_{0}\right) \propto 1, B_{0}=100$ & 0.0040 & $\mathbf{1 . 0 0 0 0}$ & 0.0009 & 27.9 \\
\hline$b=10^{-3}$ & 0.2507 & $\mathbf{1 . 0 0 0 0}$ & 0.2400 & 25.5 \\
$b=10^{-4}$ & 0.0818 & $\mathbf{1 . 0 0 0 0}$ & 0.0863 & 25.5 \\
$b=10^{-5}$ & 0.0339 & $\mathbf{1 . 0 0 0 0}$ & 0.0349 & 25.3 \\
\hline
\end{tabular}



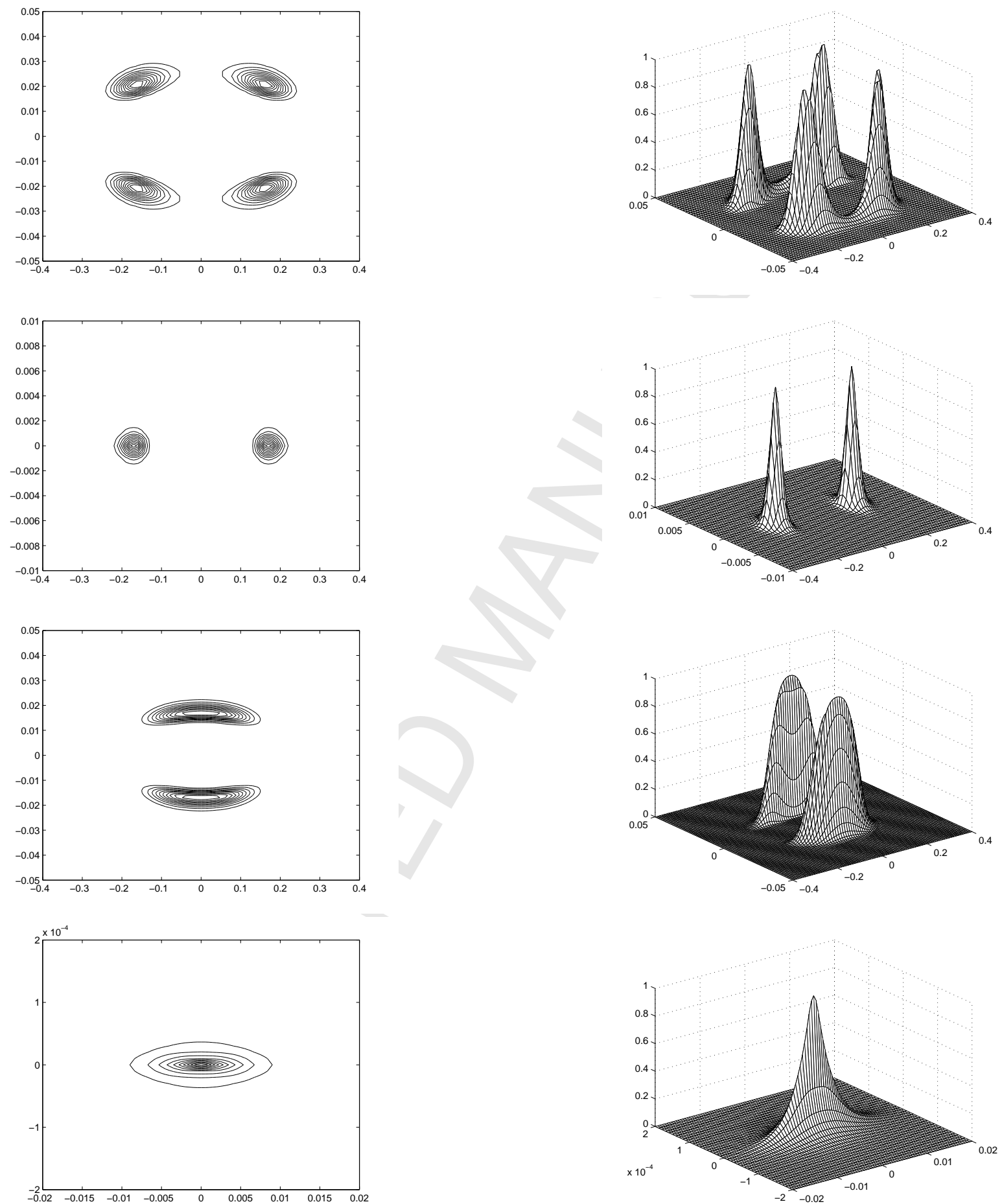

Figure 1: Contour and surface plots of the (scaled) profile likelihood $l\left(\sqrt{\theta_{1}}, \sqrt{\theta_{2}}\right) / \max \left(l\left(\sqrt{\theta_{1}}, \sqrt{\theta_{2}}\right)\right)$, where $l\left(\sqrt{\theta_{1}}, \sqrt{\theta_{2}}\right)=p\left(\mathbf{y} \mid \sqrt{\theta_{1}}, \sqrt{\theta_{2}}, \sigma_{\varepsilon}^{2 \text { tr }}, \mu_{0}^{\mathrm{tr}}, a_{0}^{\mathrm{tr}}\right)$ for simulated data with $\left(\theta_{1}^{\operatorname{tr}}, \theta_{2}^{\text {tr }}\right)=\left(0.15^{2}, 0.02^{2}\right)$ (first row), $\left(\theta_{1}^{\operatorname{tr}}, \theta_{2}^{\text {tr }}\right)=\left(0.15^{2}, 0\right)$ ( second row), $\left(\theta_{1}^{\operatorname{tr}}, \theta_{2}^{\text {tr }}\right)=\left(0,0.02^{2}\right)$ (third row), and $\left(\theta_{1}^{\operatorname{tr}}, \theta_{2}^{\operatorname{tr}}\right)=(0,0)$ (last row) 
(a)

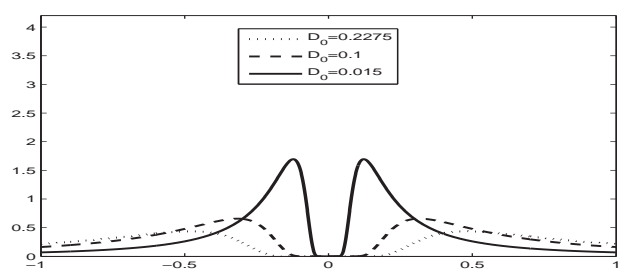

(c)

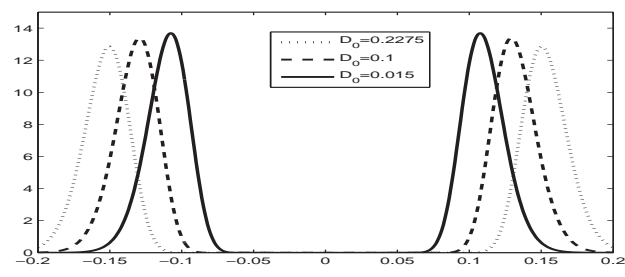

(e)

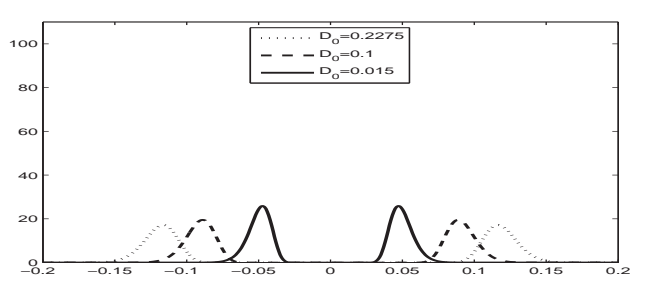

(b)

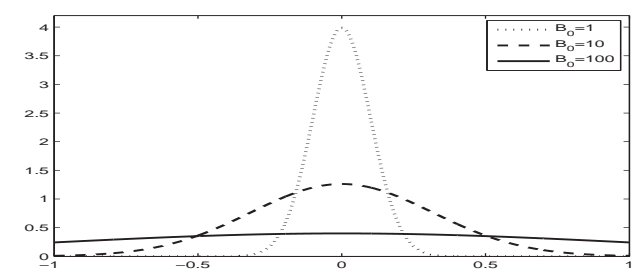

(d)

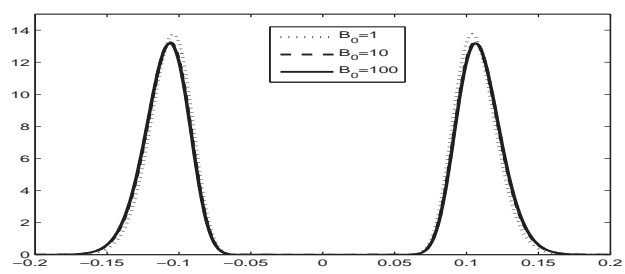

(f)

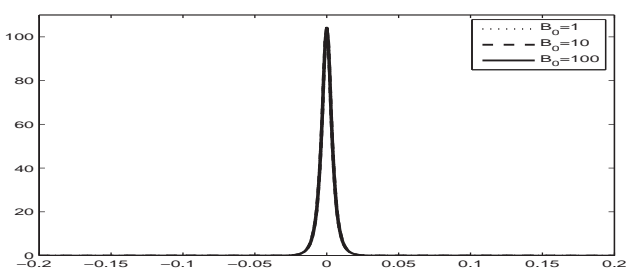

Figure 2: Prior and posterior densities for $\pm \sqrt{\theta_{1}}$ under the prior distributions $\theta_{1} \sim$ $\mathcal{G}^{-1}\left(0.5, D_{0}\right)$ (left hand side) and $\pm \sqrt{\theta_{1}} \sim \mathcal{N}\left(0, B_{0} \sigma_{\varepsilon}^{2}\right)$ (right hand side). Top: priors, middle: posterior, if true value of $\theta_{1}$ is equal to $\theta_{1}^{\text {tr }}=0.01$; bottom: posterior, if true value of $\theta_{1}$ is equal to $\theta_{1}^{\text {tr }}=0$ 
(a)

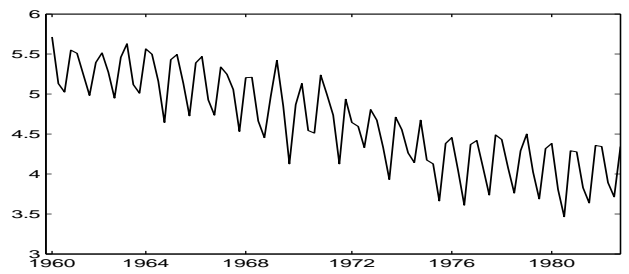

(c)

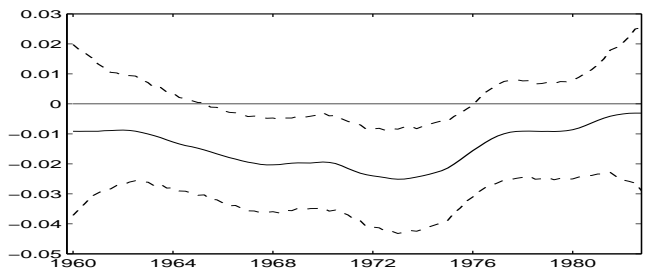

(b)

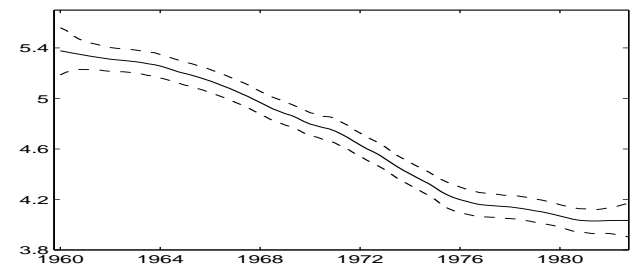

(d)

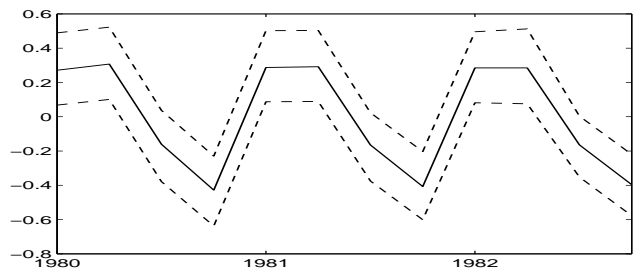

Figure 3: UK coal consumption; (a) observations 1/1960 to 4/1982 (log scale), posterior means and point-wise $95 \%$ credible regions of (b) the level $\mu_{t},(\mathrm{c})$ the drift $a_{t}$ and (d) the seasonal component $s_{t}$ in the last three years under the centered parameterization
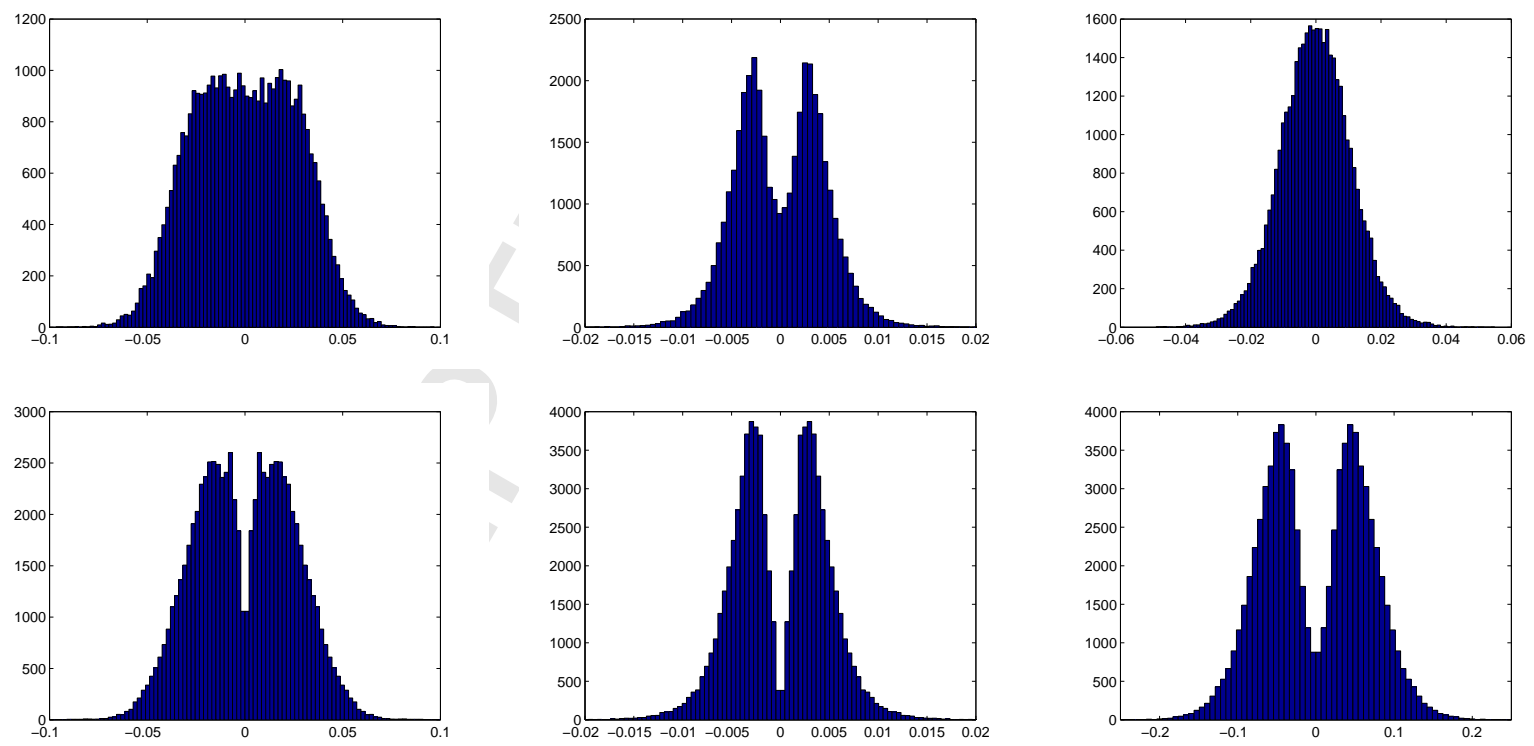

Figure 4: UK coal consumption; posterior densities of $\pm \sqrt{\theta_{1}}$ (left), $\pm \sqrt{\theta_{2}}$ (middle) and $\pm \sqrt{\theta_{3}}$ (right) estimated from the MCMC draws under different priors; top: $\mathcal{N}(0,1)$ prior for $\pm \sqrt{\theta_{i}}$; bottom: $\mathcal{G}^{-1}\left(-0.5,10^{-7}\right)$-prior for $\theta_{i}$ 

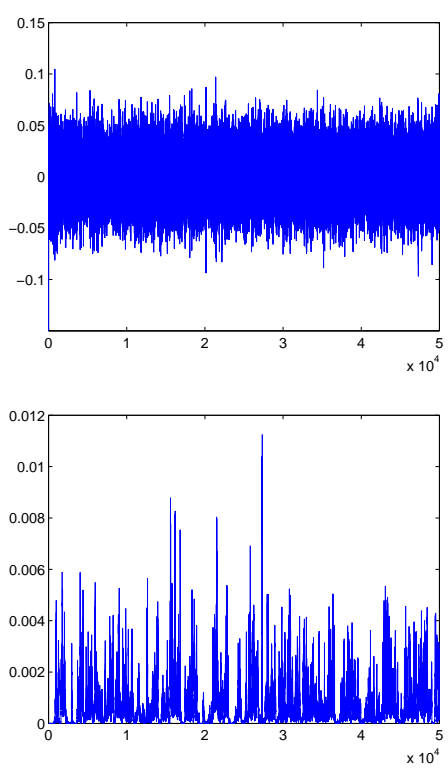
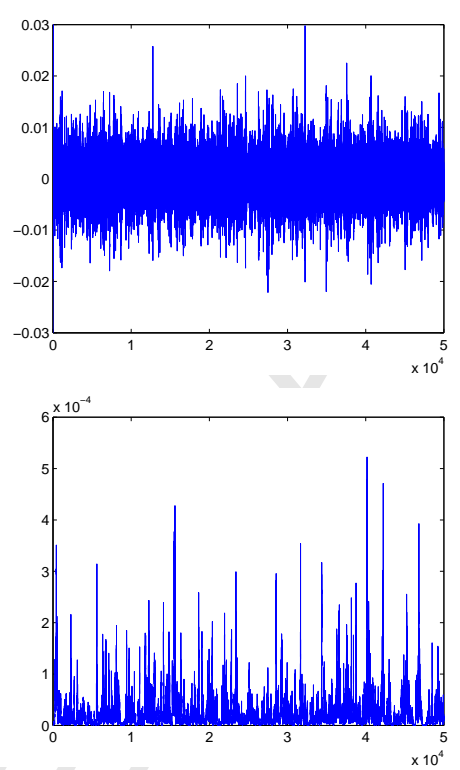
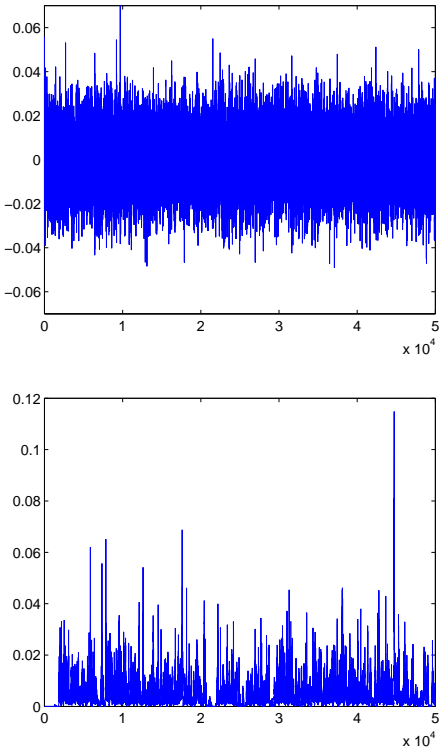

Figure 5: UK coal consumption; MCMC draws for $\pm \sqrt{\theta_{1}}$ (left), $\pm \sqrt{\theta_{2}}$ (middle) and $\pm \sqrt{\theta_{3}}$ (right) under different parameterizations; top: noncentered parameterization with a $\mathcal{N}(0,1)$-prior for $\pm \sqrt{\theta_{i}}$; bottom: centered parameterization with a $\mathcal{G}^{-1}\left(-0.5,10^{-7}\right)$ prior for $\theta_{i}$ 

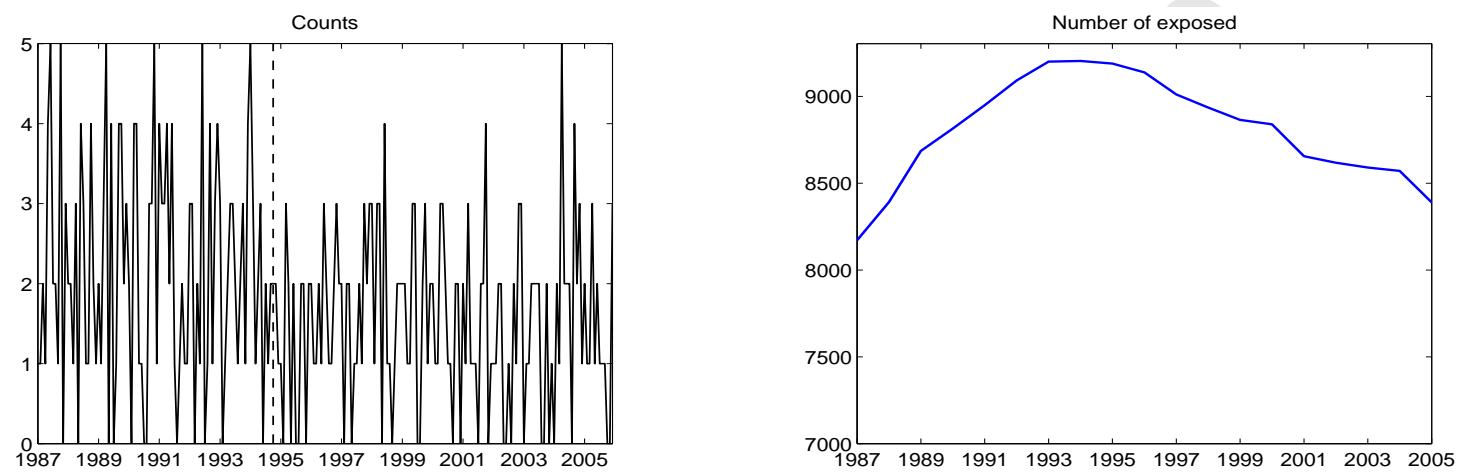

Figure 6: Road safety data; (a) counts of killed or injured children, (b) number of children exposed
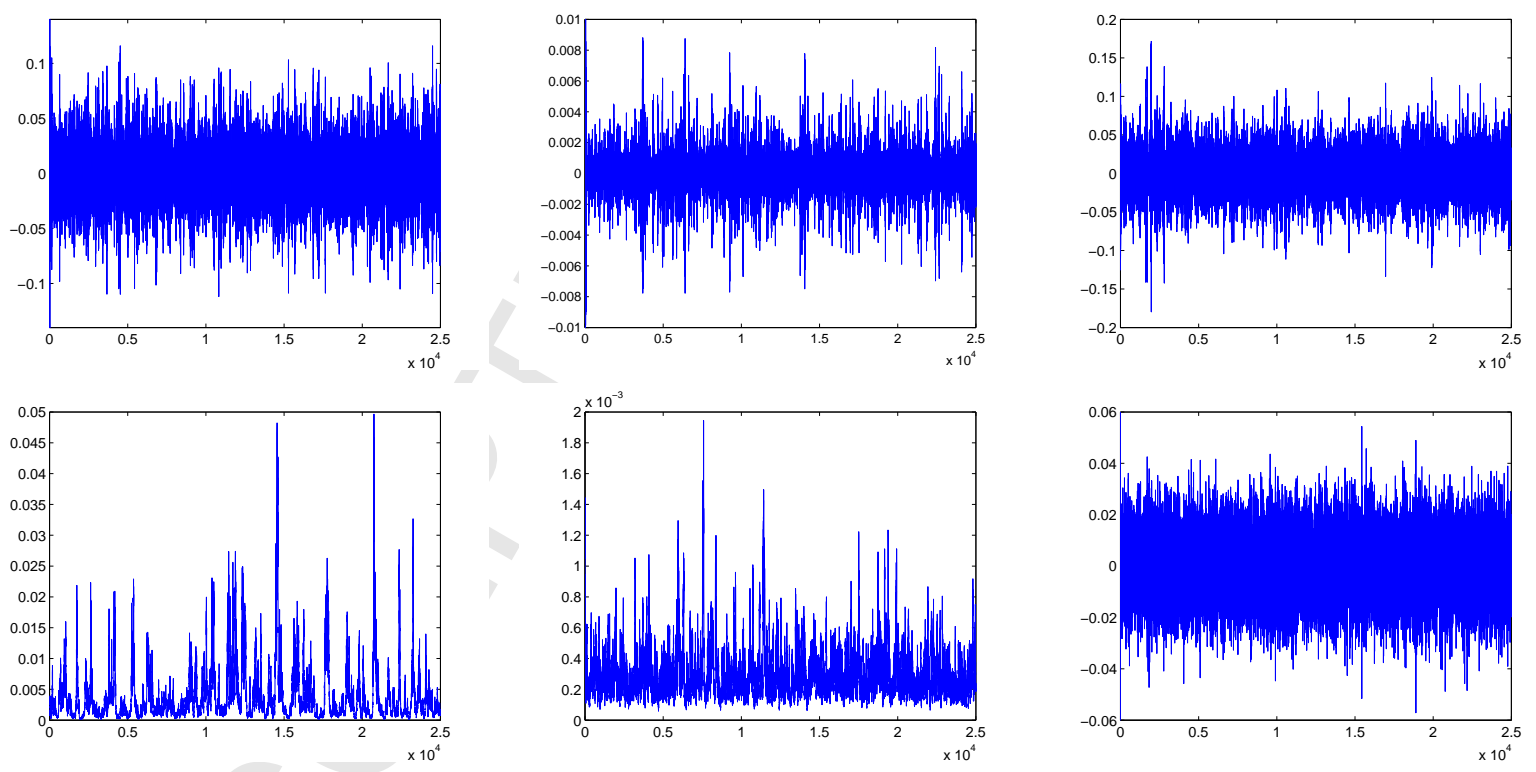

Figure 7: Road safety data; top: MCMC draws for $\pm \sqrt{\theta_{1}}$ (left), $\pm \sqrt{\theta_{2}}$ (middle) and $\pm \sqrt{\theta_{3}}$ (right) for the noncentered parameterization; bottom: MCMC draws for $\theta_{1}$ (left), $\theta_{2}$ (middle) and $\pm \sqrt{\theta_{3}}$ (right) under the partially noncentered parameterization 

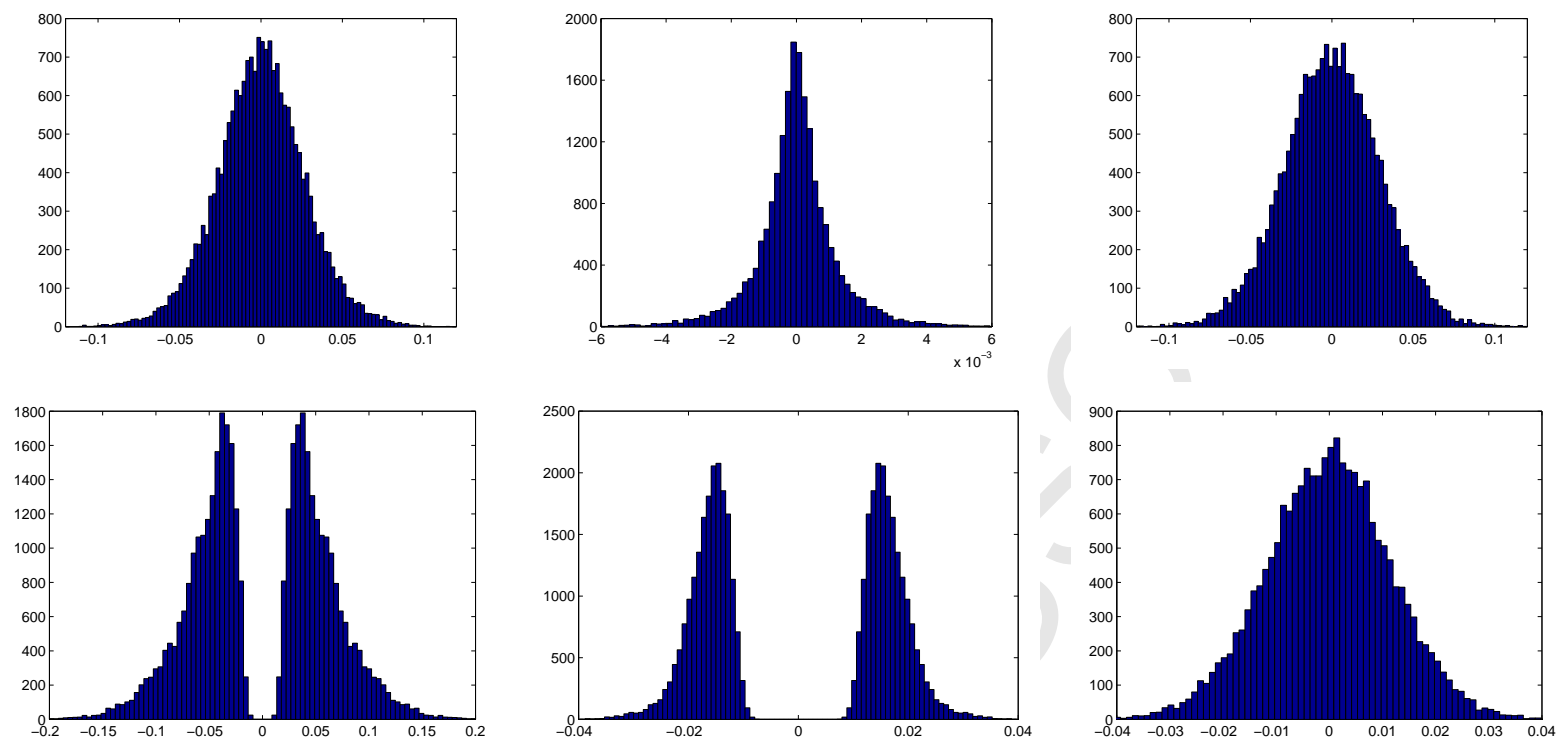

Figure 8: Road safety data; histograms of the MCMC draws for $\pm \sqrt{\theta_{1}}$ (left), $\pm \sqrt{\theta_{2}}$ (middle) and $\pm \sqrt{\theta_{3}}$ (right); top: $\mathcal{N}(0,1)$ prior for $\pm \sqrt{\theta_{i}}, i=1,2,3$; bottom: $\mathcal{G}^{-1}(0.1,0.001)$ prior for $\theta_{1}$ and $\theta_{2}, \mathcal{N}(0,1)$ prior for $\pm \sqrt{\theta_{3}}$

(a)

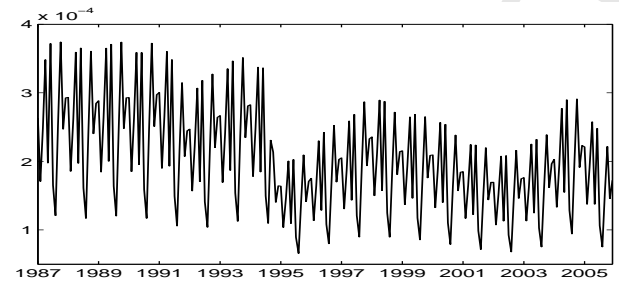

(c)

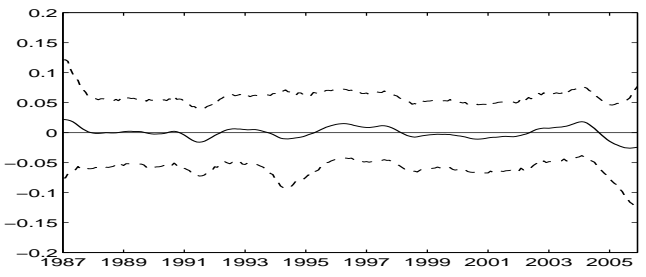

(b)

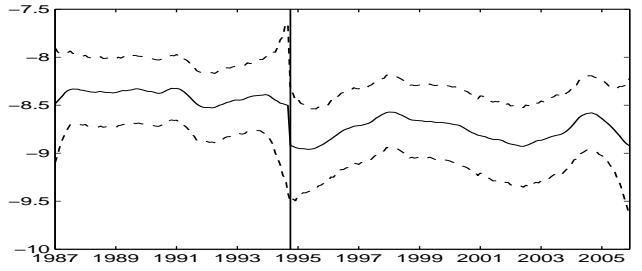

(d)

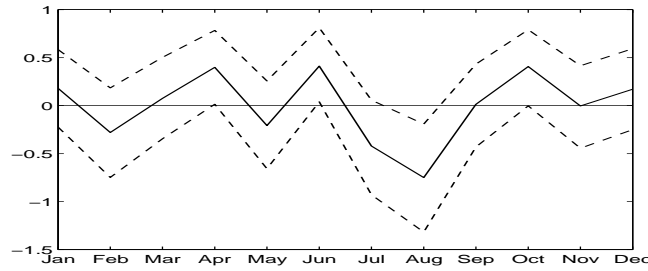

Figure 9: Road safety data; (a) posterior mean of the risk $\lambda_{t}$; posterior means and pointwise $95 \%$ credible regions of (b) the level $\mu_{t}$, (c) the drift $a_{t}$ and (d) the seasonal component $s_{t}$ in year 2005 under the centered parameterization 

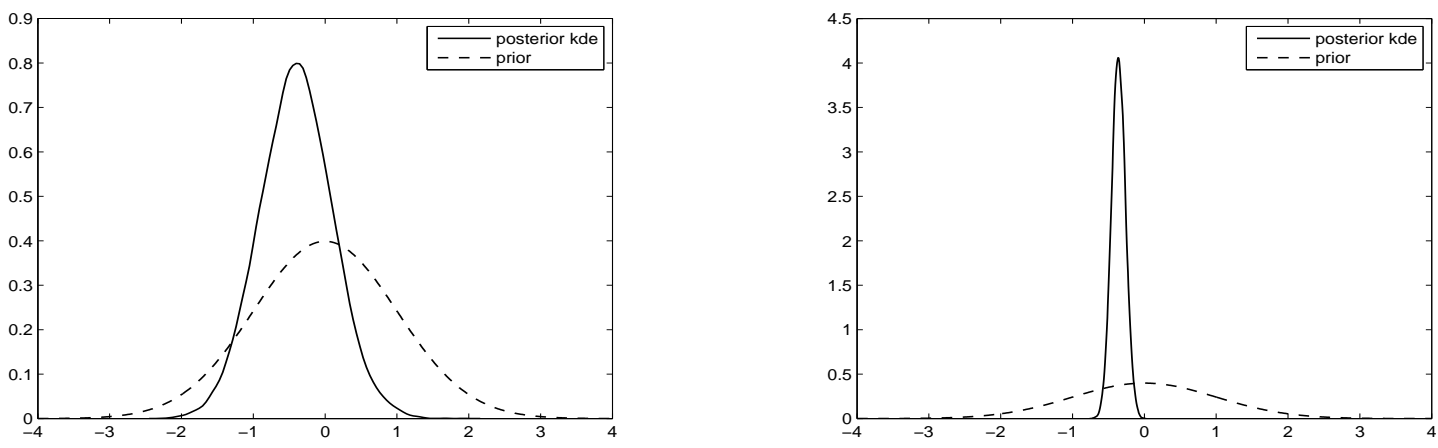

Figure 10: Road safety data; posterior density of the intervention effect $\Delta$ in comparison to the prior; left: unrestricted basic structural model, right: Poisson regression model with seasonal pattern (selected model)

(a)

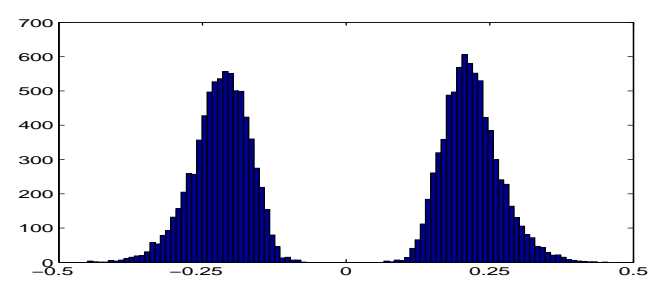

(c)

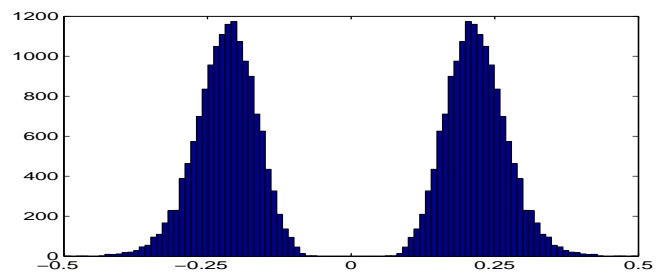

(b)

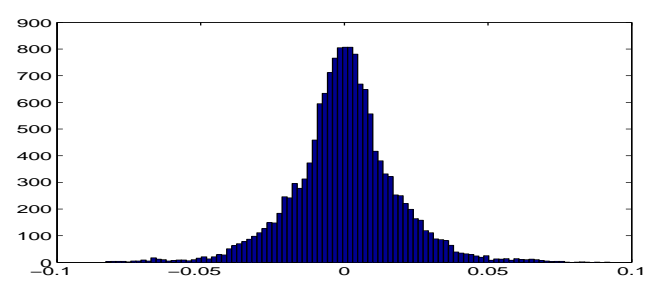

$(d)$

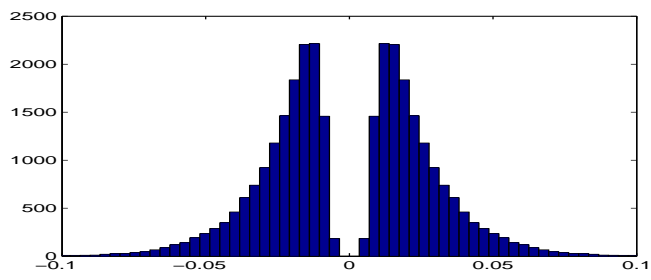

Figure 11: Purse snatching data; histograms for $\pm \sqrt{\theta_{1}}$ (left) and $\pm \sqrt{\theta_{2}}$ (right); top: $\mathcal{N}(0,1)$ prior for $\pm \sqrt{\theta_{1}}$ and $\pm \sqrt{\theta_{2}}$; bottom: $\mathcal{G}^{-1}(-0.5,0.0001)$-prior for $\theta_{1}$ and $\theta_{2}$ 